\title{
Tail asymptotics of the Brownian signature
}

Article

Accepted Version

Boedihardjo, H. and Geng, X. (2019) Tail asymptotics of the Brownian signature. Transactions of the American

Mathematical Society, 372. pp. 585-614. ISSN 1088-6850 doi: https://doi.org/10.1090/tran/7683 Available at https://centaur.reading.ac.uk/78800/

It is advisable to refer to the publisher's version if you intend to cite from the work. See Guidance on citing.

To link to this article DOI: http://dx.doi.org/10.1090/tran/7683

Publisher: American Mathematical Society

All outputs in CentAUR are protected by Intellectual Property Rights law, including copyright law. Copyright and IPR is retained by the creators or other copyright holders. Terms and conditions for use of this material are defined in the End User Agreement.

www.reading.ac.uk/centaur

\section{CentAUR}

Central Archive at the University of Reading

Reading's research outputs online 


\title{
Tail Asymptotics of the Brownian Signature
}

\author{
H. Boedihardjo, X. Geng ${ }^{\dagger}$
}

\begin{abstract}
The signature of a path $\gamma$ is a sequence whose $n$-th term is the order- $n$ iterated integrals of $\gamma$. It arises from solving multidimensional linear differential equations driven by $\gamma$. We are interested in relating the path properties of $\gamma$ with its signature. If $\gamma$ is $C^{1}$, then an elegant formula of Hambly and Lyons relates the length of $\gamma$ to the tail asymptotics of the signature. We show an analogous formula for the multidimensional Brownian motion, with the quadratic variation playing a similar role to the length. In the proof, we study the hyperbolic development of Brownian motion and also obtain a new subadditive estimate for the asymptotic of signature, which may be of independent interest. As a corollary, we strengthen the existing uniqueness results for the signatures of Brownian motion.
\end{abstract}

\section{Introduction}

\subsection{Path driven differential equations and iterated integrals}

Path-driven differential equations of the form

$$
d Y_{t}=\sum_{i=1}^{d} A_{i} Y_{t} \mathrm{~d} \gamma_{t}^{i}, Y_{0}=y
$$

where $\gamma=[0, T] \rightarrow \mathbb{R}^{d}, \gamma=\left(\gamma^{1}, \ldots, \gamma^{d}\right)$ and $A_{i}: \mathbb{R}^{n} \rightarrow \mathbb{R}^{n}$ is linear, has a Taylor expansion of the form

$$
Y_{T}=\sum_{n=0}^{\infty} \sum_{1 \leq i_{1}, \ldots, i_{n} \leq d} A_{i_{n}} A_{i_{n-1}} \ldots A_{i_{1}} \int_{0}^{T} \ldots \int_{0}^{t_{2}} \mathrm{~d} \gamma_{t_{1}}^{i_{1}} \ldots \mathrm{d} \gamma_{t_{n}}^{i_{n}} .
$$

${ }^{*}$ Department of Mathematics and Statistics , University of Reading, Reading RG6 6AX, United Kingdom. Email: h.s.boedihardjo@reading.ac.uk.

${ }^{\dagger}$ Department of Mathematical Sciences, Carnegie Mellon University, Pittsburgh PA 15213, United States. Email: xig@andrew.cmu.edu. 
In particular, $Y_{t}$ is a linear function of the signature of $\gamma$ on $[0, T]$ (also known as the Chen series [8]), defined as

$$
g \triangleq\left\{\int_{0<t_{1}<\cdots<t_{n}<T} \mathrm{~d} \gamma_{t_{1}} \otimes \cdots \otimes \mathrm{d} \gamma_{t_{n}}: n \in \mathbb{N}\right\} .
$$

If $\gamma$ is a stochastic process, then the map from $g$ to $Y_{T}$ is a deterministic map (independent of the sample path). Some useful properties about $Y_{T}$ can be deduced from $S(\gamma)_{0, T}$ through the Taylor expansion (1.2). As the simplest example, if $g$ is well-defined almost surely, then so would $Y_{T}$, with the exceptional set being independent of $A$.

Motivated by the use of signature in solving differential equations, the signature of $\gamma$ has been used to store information about the path $\gamma$ for the purpose of e.g. handwriting recognition ([23]). Many of these signature-based methods would benefit from a better understanding of how the signature is related to the geometric properties of $\gamma$. For instance, certain functionals of signature may contain more useful information about handwriting recognition than others, in which case we may save computational time by focusing on these features. The "reconstruction problem" of a path from its signature has attracted interests recently in [7], [11], [18], [19], [22].

In the rough path literature, the first main result in this direction was due to Hambly and Lyons [12] that every continuous path with bounded variation is uniquely determined by its signature up to a tree-like equivalence. Loosely speaking, two paths are tree-like equivalence if one can be obtained by adding tree-like pieces to the other, see figure below.

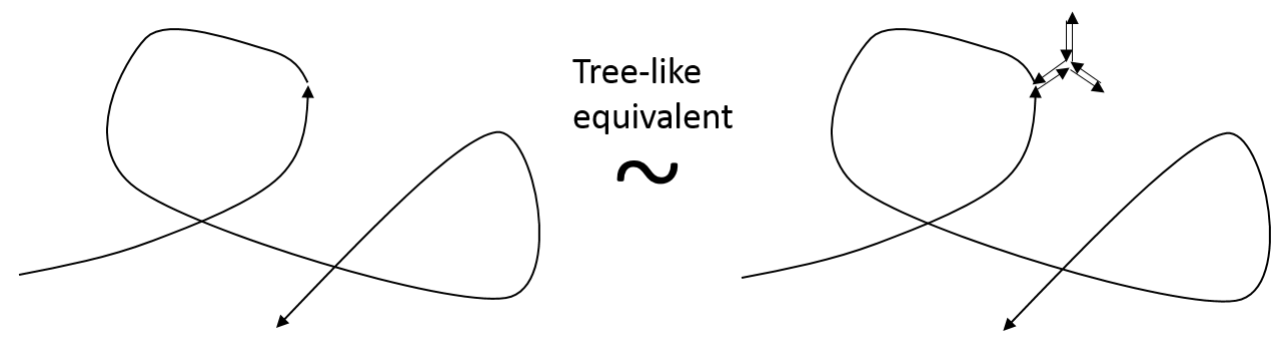

As we do not need the precise definition of tree-like in this paper, we refer the interested readers to [12]. Hambly-Lyons' uniqueness result was extended to the general rough path case in [3].

Along with the uniqueness results mentioned above, it was also shown ([3], [12]) that every tree-like equivalence class contains a unique representative path $\gamma$ which does not contain any tree-like pieces. This representative path is called the tree-reduced path. On the other hand, signatures have a certain algebraic 
structure (see Theorem 2.15 in [6]) which ensures that every term of a signature element $g$ can be recovered from looking at the tail of $g$. Therefore, it is natural and reasonable to expect that some intrinsic geometric properties associated with a tree-reduced rough path can be explicitly recovered from the tail behavior of its signature.

In the bounded variation case, it was proved that the length of a path $\gamma$ can be recovered from the tail asymptotics of its signature $g$ in the following way:

$$
\|\gamma\|_{1-\mathrm{var}}=\lim _{n \rightarrow \infty}\left(n !\left\|g_{n}\right\|_{\text {proj }}\right)^{\frac{1}{n}}
$$

provided that $\gamma \in C^{1}$ when parametrized by unit speed and the modulus of continuity $\delta_{\gamma^{\prime}}$ for $\gamma^{\prime}$ satisfies $\delta_{\gamma^{\prime}}(\varepsilon)=o\left(\varepsilon^{3 / 4}\right)$ as $\varepsilon \downarrow 0$. Here $g_{n}$ is the $n$-th term of the signature $g$ and the tensor norm is the projective norm induced by the Euclidean norm on $\mathbb{R}^{d}$ (see Definition 2.2). The notation $\|\gamma\|_{1-v a r}$ denote the 1-variation of $\gamma$ which is the same as the length of $\gamma$. This formula (1.4) now also holds for general $C^{1}$ paths [18], piecewise linear paths and monotonely increasing paths. Note that in dimension 1, the assumption that $\gamma \in C^{1}$ with respect to the unit speed parametrization implies that $\gamma$ is monotonic in all coordinates. Therefore (1.4) is only interesting when the dimension is greater than 1. It has been conjectured that the same result should hold for all tree-reduced continuous path with bounded variation. However, very little progress has been made towards a complete solution.

For an arbitrary continuous path with bounded variation $\gamma$, one can easily see that

$$
\left\|g_{n}\right\|_{\text {proj }} \leqslant \frac{\|\gamma\|_{1-\text { var }}^{n}}{n !}, \quad \forall n \in \mathbb{N} .
$$

So the length conjecture (1.4) is about establishing a matching lower bound. If proved to be true in general, it will indicate that for a tree-reduced path, the signature components decay in an exact factorial rate. The original idea of Hambly and Lyons for proving (1.4) in the $C^{1}$-case is looking at the lifting $X^{\lambda}$ of $\lambda \cdot \gamma$ (rescaling $\gamma$ by a large constant $\lambda$ ) to the hyperbolic manifold of constant curvature -1 (the hyperbolic development). It turns out that when $\lambda \rightarrow \infty, X^{\lambda}$ becomes more and more like a hyperbolic geodesic in the sense that the hyperbolic distance between the two endpoints of $X^{\lambda}$ is asymptotically comparable to its hyperbolic length. As a simple consequence of the nature of hyperbolic development, the said hyperbolic distance is related to the signature of $\gamma$ is a fairly explicit way, while the hyperbolic length is the same as the original length. In this way, one sees a lower bound for the signature in terms of the length. It seems to us that in the deterministic setting, the technique of hyperbolic development is essentially a 
$C^{1}$-technique which requires major modification in the general bounded variation case in quite a fundamental way.

In parallel, we could certainly ask a similar question in the rough path context. According to Lyons [15], for a rough path $\mathbf{X}$ with finite $p$-variation $(p \geqslant 1$, see Definition 2.3) the signature estimate takes the form

$$
\left\|g_{n}\right\|_{\text {proj }} \leqslant \frac{\omega(\mathbf{X})^{\frac{n}{p}}}{\left(\frac{n}{p}\right) !}, \quad \forall n \in \mathbb{N},
$$

where $\omega(\mathbf{X})$ is a constant depending on the $p$-variation of $\mathbf{X}$ and $\left(\frac{n}{p}\right) !=\Gamma\left(\frac{n}{p}+1\right)$ with $\Gamma$ being the gamma function. To expect an analogue of (1.4) for rough paths (what this actually means is not even clear at at this point), it is natural to search lower bounds for $g_{n}$ of the same form and look at the quantity

$$
\tilde{L}_{p} \triangleq \limsup _{n \rightarrow \infty}\left(\left(\frac{n}{p}\right) !\left\|g_{n}\right\|_{\text {proj }}\right)^{\frac{p}{n}} .
$$

On the one hand, the reason of looking at the "limsup" instead of an actual limit is that, unlike the bounded variation case, the limit does not generally exist for rough paths. For instance, one could easily find examples of tree-reduced geometric rough paths with infinitely many zero signature terms (for instance $\mathbf{X}_{t} \triangleq \exp (t[v, w])$ for certain vectors $\left.v, w \in \mathbb{R}^{d}\right)$. One might expect that $\widetilde{L}_{p}$ is equal to the $p$-variation of the underlying rough path. However, this cannot be the case since $\widetilde{L}_{p}=0$ for a bounded variation path when $p>1$ due to (1.5), whereas bounded variation paths have non-zero $p$-variation. On the other hand, if we define the "local $p$-variation" of a rough path in the same way as the usual $p$-variation but additionally by requiring that the mesh size of partitions goes to zero, it is easy to see that the local $p$-variation of a bounded variation path is also zero when $p>1$. Therefore, it is not entirely unreasonable to expect that the quantity $\widetilde{L}_{p}$ recovers the local $p$-variation of $\mathbf{X}$.

In the present article, we investigate a similar problem for the Brownian rough path $\mathbf{B}_{t}$, which is the canonical lifting of the Brownian motion $B_{t}$ as geometric $p$ rough paths for $2<p<3$. One can equivalently view it as the Brownian motion coupled with the Lévy area process. It is well-known that $B_{t}$ has a quadratic variation process, which can be viewed as the local 2-variation of Brownian motion in certain probabilistic sense. In view of the previous discussion, if we define the normalized "limsup"

$$
\widetilde{L}_{s, t} \triangleq \limsup _{n \rightarrow \infty}\left(\left(\frac{n}{2}\right) !\left\|\int_{s<t_{1}<\cdots<t_{n}<t} \operatorname{od} B_{t_{1}} \otimes \cdots \otimes \operatorname{od} B_{t_{n}}\right\|\right)^{\frac{2}{n}}
$$


(od means the Stratonovich integral) for the Brownian signature path under suitable tensor norms, one might expect that $\widetilde{L}_{s, t}$ recovers some sort of quadratic variation of the Brownian rough path. The aim of the present article is to establish a result of this kind. Even with Lyons' estimate (1.6), it is a priori unclear that $\widetilde{L}_{s, t}$ is even finite since Brownian motion has infinite 2-variation almost surely.

We are going to show that $\widetilde{L}_{s, t}$ is a deterministic multiple of $t-s: \widetilde{L}_{s, t}=\kappa(t-s)$ for some deterministic constant $\kappa$. This implies that the natural speed of Brownian motion (i.e. its quadratic variation) can be recovered from the tail asymptotics of its signature. In addition, we establish upper and lower bounds on the constant $\kappa$.

On the one hand, the upper estimate is shown by using general rough path arguments and does not reflect the tree-reduced nature of the Brownian rough path at all. The deterministic nature of $\widetilde{L}_{s, t}$ comes from the fact that Brownian motion has independent increments. The result holds under a wide choice of tensor norms.

On the other hand, the lower estimate is obtained by considering the hyperbolic development of Brownian motion. Our calculation diverges early on from the work of Hambly and Lyons [12] for the bounded variation paths, as we make use of martingale arguments instead of deterministic hyperbolic analysis. Our lower estimate allows us to conclude that the Brownian rough path is tree-reduced with probability one and also its natural parametrization can be recovered from the tail asymptotics of the Brownian signature. In particular, with probability one, every Brownian rough path is uniquely determined by its signature. This result is stronger than the existing uniqueness results for Brownian motion in the literature (c.f. [2], [14]), since it was only known that the signature determines the Brownian rough path up to reparametrization.

Our main result on the upper and lower estimates of $\widetilde{L}_{s, t}$ can be summarized as follows.

Theorem 1.1. Let $B_{t}=\left(B_{t}^{1}, \cdots, B_{t}^{d}\right)$ be a d-dimensional Brownian motion $(d \geqslant$ 2). Define $\widetilde{L}_{s, t}$ by

$$
\widetilde{L}_{s, t} \triangleq \limsup _{n \rightarrow \infty}\left(\left(\frac{n}{2}\right) !\left\|\int_{s<t_{1}<\cdots<t_{n}<t} \operatorname{od} B_{t_{1}} \otimes \cdots \otimes \circ \mathrm{od} B_{t_{n}}\right\|\right)^{\frac{2}{n}}
$$

where $\|\cdot\|$ is an admissible norm (see Definition 2.1).

(1) (upper estimate) If each element of the canonical basis $\left\{\mathrm{e}_{1}, \cdots, \mathrm{e}_{d}\right\}$ of $\mathbb{R}^{d}$ has norm one with respect to $\|\cdot\|$, then there exists a deterministic constant 
$\kappa_{d} \leqslant d^{2}$ depending on the choice of tensor norms, such that with probability one

$$
\widetilde{L}_{s, t}=\kappa_{d}(t-s) \quad \forall s<t .
$$

(2) (lower estimate) Under the $l^{p}$-norm $(1 \leqslant p \leqslant 2)$ on $\mathbb{R}^{d}$ and the associated projective tensor norms on the tensor products, we have

$$
\kappa_{d} \geqslant \frac{d-1}{2} .
$$

Remark 1.1. The one dimensional case $(d=1)$ is uninteresting and the result of Theorem 1.1 holds trivially since $\widetilde{L}_{s, t} \equiv 0$ in this case.

The relation between the iterated integrals and the geometry of $\gamma$ are generally complex and it is therefore somewhat surprising that a simple formula exists relating between the quadratic variation and length with the norms of the signature.

There is a number of related problems to Theorem 1.1. It is not known whether (1.9) remains true if we replace the limsup with lim, nor do we know the exact value of $\kappa_{d}$. Perhaps the biggest open problem of all, is how to generalise both (1.9) and Hambly-Lyons' formula (1.4) to rough paths, though this is known to be difficult even for (non- $C^{1}$ ) bounded variation paths. We will elaborate more on the open problems and their ramifications in Section 6.

Our article is organized in the following way. In Section 2, we present some basic notions from rough path theory which are needed for our analysis. In Section 3, we prove the first part of Theorem 1.1. In Section 4, we prove the second part of Theorem 1.1. In that section we also present some crucial details for understanding the hyperbolic development which seems to be incomplete or missing in the literature. In Section 5, we present some interesting applications of our main result to the Brownian rough path itself. In Section 6, we give some concluding remarks and discuss a few related further problems.

\section{Notions from rough path theory}

In this section, we present some basic notions from rough path theory which are needed for our study. Although our main result concerns solely about Brownian motion, key sections of our argument holds generally for rough paths and is viewed most naturally in that context. We refer the reader to the monographs [6], [10], [16] for a systematic introduction on rough path theory.

Suppose that $V$ is a finite dimensional normed vector space. For each $n \in \mathbb{N}$, define $T^{(n)}(V) \triangleq \oplus_{i=0}^{n} V^{\otimes i}$, and let $T((V))$ be the algebra of formal sequences of homogeneous tensors $a=\left(a_{0}, a_{1}, a_{2}, \cdots\right)$ with $a_{n} \in V^{\otimes n}$ for each $n$. 
Definition 2.1. A family of tensor norms $\left\{\|\cdot\|_{V^{\otimes n}}: n \geqslant 1\right\}$ on the tensor products is called admissible if

(1) for any $a \in V^{\otimes m}$ and $b \in V^{\otimes n}$,

$$
\|a \otimes b\|_{V^{\otimes(m+n)}} \leqslant\|a\|_{V_{\otimes m}}\|b\|_{V^{\otimes n}}
$$

(2) for any permutation $\sigma$ on $\{1, \ldots, n\}$ and $a \in V^{\otimes n}$,

$$
\left\|\mathcal{P}^{\sigma}(a)\right\|_{V \otimes n}=\|a\|_{V \otimes n}
$$

where $\mathcal{P}^{\sigma}$ is the linear operator on $V^{\otimes n}$ induced by $a_{1} \otimes \cdots \otimes a_{n} \mapsto a_{\sigma(1)} \otimes \cdots \otimes a_{\sigma(n)}$ for $a_{1}, \cdots, a_{n} \in V$.

We call it a family of cross-norms if the inequality in (2.1) is an equality.

Definition 2.2. The projective tensor norm on $V^{\otimes n}$ is defined to be

$$
\|a\|_{\text {proj }} \triangleq \inf \left\{\sum_{l}\left|a_{1}^{(l)}\right| \cdots\left|a_{n}^{(l)}\right|: \text { if } a=\sum_{l} a_{1}^{(l)} \otimes \cdots \otimes a_{n}^{(l)}\right\} \text {. }
$$

It is known that the projective tensor norm is the largest cross-norm on $V^{\otimes n}$. In the case when $V=\mathbb{R}^{d}$ is equipped with the $l^{1}$-norm, one can see by definition that the projective tensor norm on $V^{\otimes n}$ is just the $l^{1}$-norm under the canonical tensor basis induced from the one on $\mathbb{R}^{d}$.

We assume that $V$ is equipped with a family of admissible tensor norms. Define $\triangle \triangleq\{(s, t): 0 \leqslant s \leqslant t \leqslant 1\}$. Given $p \geqslant 1$, we denote $\lfloor p\rfloor$ as the largest integer not exceeding $p$.

Definition 2.3. A multiplicative functional of degree $n \in \mathbb{N}$ is a continuous map $\mathbf{X}_{., .}=\left(1, \mathbb{X}_{., \cdot}^{1}, \cdots, \mathbb{X}_{., \cdot}^{n}\right): \triangle \rightarrow T^{(n)}(V)$ which satisfies

$$
\mathbf{X}_{s, u} \otimes \mathbf{X}_{u, t}=\mathbf{X}_{s, t}, \text { for } 0 \leqslant s \leqslant u \leqslant t \leqslant 1
$$

Let $\mathbf{X}, \mathbf{Y}$ be two multiplicative functionals of degree $n$. Define

$$
d_{p}(\mathbf{X}, \mathbf{Y}) \triangleq \max _{1 \leqslant i \leqslant n} \sup _{\mathcal{P}}\left(\sum_{l}\left\|\mathbb{X}_{t_{l-1}, t_{l}}^{i}-\mathbb{Y}_{t_{l-1}, t_{l}}^{i}\right\|_{V^{\otimes i}}^{\frac{p}{i}}\right)^{\frac{i}{p}}
$$

where the supremum is taken over all possible finite partitions $\mathcal{P}=\left(t_{0}<t_{1}<\right.$ $\left.\ldots<t_{n}\right)$ of $[0,1] . d_{p}$ is called the p-variation metric. If $d_{p}(\mathbf{X}, \mathbf{1})<\infty$ where $\mathbf{1}=(1,0, \cdots, 0)$, we say that $\mathbf{X}$ has finite $p$-variation. A multiplicative functional of degree $\lfloor p\rfloor$ with finite $p$-variation is called a $p$-rough path. 
The following important result, proved by Lyons [15], asserts that "iterated path integrals" for a rough path are also well defined.

Theorem 2.1. [Lyons' extension theorem] Let $\mathbf{X}=\left(1, \mathbb{X}^{1}, \cdots, \mathbb{X}^{\lfloor p\rfloor}\right)$ be a $p$ rough path. Then for any $n \geqslant\lfloor p\rfloor+1$, there exists a unique continuous map $\mathbb{X}^{n}: \Delta \rightarrow V^{\otimes n}$, such that

$$
\mathbb{X}_{., \cdot} \triangleq\left(1, \mathbb{X}_{., \cdot}^{1}, \cdots, \mathbb{X}_{., \cdot}^{\lfloor p\rfloor}, \cdots, \mathbb{X}_{., \cdot}^{n}, \cdots\right)
$$

is a multiplicative functional in $T((V))$ whose projection onto $T^{(n)}(V)$ has finite p-variation for every $n$.

Remark 2.1. Due to the multiplicative structure, when we consider a rough path, one could simply look at the path $t \mapsto \mathbf{X}_{0, t}$ whose increments are defined to be $\mathbf{X}_{s}^{-1} \otimes \mathbf{X}_{t}$.

Remark 2.2. When $p=1$ and $\mathbf{X}$ is a continuous path with bounded variation, all the previous notions reduces to the classical iterated path integrals defined in the sense of Lebesgue-Stieltjes.

Definition 2.4. Let $\mathbf{X}$ be a $p$-rough path. The path $t \mapsto \mathbb{X}_{0, t} \in T((V))$ defined by Lyons' extension theorem is called the signature path of $\mathbf{X}$. The quantity $\mathbb{X}_{0,1}$ is called the signature of $\mathbf{X}$.

Among general rough paths there is a fundamental class of paths called geometric rough paths.

Definition 2.5. For a continuous path with bounded variation $\gamma:[0,1] \rightarrow V$, define

$$
\mathbb{X}_{s, t}^{n}=\int_{s<u_{1}<\cdots<u_{n}<t} \mathrm{~d} \gamma_{u_{1}} \otimes \cdots \otimes \mathrm{d} \gamma_{u_{n}}, \quad n \geqslant 1, s \leqslant t .
$$

The closure of the space

$$
\left\{\left(1, \mathbb{X}_{s, t}^{1}, \ldots, \mathbb{X}_{s, t}^{\lfloor p\rfloor}\right): \gamma \text { is a continuous path with bounded variation }\right\}
$$

under the $p$-variation metric $d_{p}$ is called the space of geometric p-rough paths.

Remark 2.3. According to [6], Theorem 2.15, the iterated integrals of bounded variation path $\gamma$ in $\mathbb{R}^{d}$ satisfies

$$
\begin{aligned}
& \int_{s<u_{1}<\cdots<u_{n}<t} \mathrm{~d} \gamma_{u_{1}}^{i_{1}} \cdots \mathrm{d} \gamma_{u_{n}}^{i_{n}} \cdot \int_{s<u_{n+1}<\cdots<u_{n+k}<t} \mathrm{~d} \gamma_{u_{n+1}}^{i_{n+1}} \cdots \mathrm{d} \gamma_{u_{n+k}}^{i_{n+k}} \\
& =\sum_{\sigma \in \mathcal{S}(n, k)} \int_{s<u_{1}<\cdots<u_{n+k}<t} \mathrm{~d} \gamma_{u_{1}}^{i_{\sigma^{-1}(1)}} \cdots \mathrm{d} \gamma_{u_{n+k}}^{i_{\sigma^{-1}(n+k)}},
\end{aligned}
$$


where - is real number multiplication and $\mathcal{S}(n, k)$ contains all permutations $\sigma$ : $\{1, \ldots, n+k\} \rightarrow\{1, \ldots, n+k\}$ such that

$$
\sigma(1)<\ldots<\sigma(n) ; \sigma(n+1)<\ldots<\sigma(n+k) .
$$

In other words, the product of $n$-th and $k$-th order iteratred integrals can be rewritten as a linear combination of $n+k$-th order iterated integrals. The property (2.2) extends to geometric rough paths. An equivalent, but coordinate invariant, formulation of $(2.2)$ is that

$$
\mathbb{X}_{s, t}^{n} \otimes \mathbb{X}_{s, t}^{k}=\sum_{\sigma \in S(n, k)} \mathcal{P}^{\sigma}\left(\mathbb{X}_{s, t}^{n+k}\right)
$$

where $\mathbb{X}^{k}$ is the $n$-th term of the signature (see Theorem 2.1), and $\mathcal{P}^{\sigma}$ is the permutation of tensors map defined in (2), Definition 2.1.

In fact, (2.3) and the multiplicative property

$$
\sum_{k=0}^{n} \mathbb{X}_{s, u}^{k} \otimes \mathbb{X}_{u, t}^{n-k}=\mathbb{X}_{s, t}^{n}
$$

are the two most fundamental algebraic properties of iterated integrals.

The space of geometric rough paths plays a fundamental role in rough path theory. In particular, a complete integration and differential equation theory with respect to geometric rough paths has been established by Lyons [15]. The rough path theory has significant applications in probability theory, mainly due to the fact that a wide class of interesting stochastic processes can be regarded as geometric rough paths in a canonical way in the sense of natural approximations.

In particular, it is known that (c.f. [21]) a multidimensional Brownian motion $B_{t}$ admits a canonical lifting as geometric $p$-rough path $\mathbf{B}_{t}$ with $p \in(2,3) . \mathbf{B}_{t}$ is called the Brownian rough path. The corresponding Brownian signature path, determined by Lyons' extension theorem, is denoted as

$$
\mathbb{B}_{s, t}=\left(1, \mathbb{B}_{s, t}^{1}, \mathbb{B}_{s, t}^{2}, \cdots\right), \quad s \leqslant t .
$$

Under the canonical tensor basis on tensor products over $V \triangleq \mathbb{R}^{d}$, for each word $\left(i_{1}, \cdots, i_{n}\right)$ over $\{1, \cdots, d\}$, the coefficient of $\mathbb{B}_{s, t}^{n}$ with respect to $\mathrm{e}_{i_{1}} \otimes \cdots \otimes \mathrm{e}_{i_{n}}$ coincides with the iterated Stratonovich integral (c.f. [6]):

$$
\mathbb{B}_{s, t}^{n ; i_{1}, \cdots, i_{n}}=\int_{s<u_{1}<\cdots<u_{n}<t} \operatorname{od} B_{u_{1}}^{i_{1}} \cdots \circ \mathrm{d} B_{u_{n}}^{i_{n}}
$$


For a given family of admissible tensor norms, we define

$$
\widetilde{L}_{s, t} \triangleq \limsup _{n \rightarrow \infty}\left(\left(\frac{n}{2}\right) !\left\|\mathbb{B}_{s, t}^{n}\right\|\right)^{\frac{2}{n}}, \quad s \leqslant t .
$$

Lyons [15] established a uniform bound for the $n$-term in the signature, $\mathbb{X}_{s, t}^{n}$, for general $p$-rough path as

$$
\left\|\mathbb{X}_{s, t}^{n}\right\| \leq \frac{\omega(\mathbf{X})^{n}}{\left(\frac{n}{p}\right) !}
$$

where $\omega(\mathbf{X})$ depends on the $p$-variation of $\mathbf{X}$. Since Brownian motion is a $p$-rough path for all $p>2$ but not $p=2$, the finiteness of $\tilde{L}_{s, t}$ does not follow from Lyons estimate (2.5). In section 3, we will establish yet another bound of iterated integrals, which is sharper than those available in the literature (e.g. [?, 1]) by a geometric factor, and holds not just for $\tilde{L}_{s . t}$ but also $\sup _{s \leq u \leq v \leq t} \tilde{L}_{u, v}$, which is essential in dealing with null sets later on.

\section{First part of the main result: the upper estimate}

In this section, we develop the proof of the first part of Theorem 1.1.

Lemma 3.1. The signature coefficients $\mathbb{B}_{s, t}^{n ; i_{1}, \cdots, i_{n}}$ satisfy the following estimate:

$$
\mathbb{E}\left[\sup _{s \leqslant u \leqslant t}\left|\mathbb{B}_{s, u}^{n ; i_{1}, \cdots, i_{n}}\right|\right] \leqslant\left(\frac{1}{2}+\sqrt{2}\right)\left(\frac{\mathrm{e}}{\sqrt{2} \pi}\right)^{\frac{1}{2}} \frac{2^{\frac{n}{2}}}{(n-2)^{\frac{1}{4}} \sqrt{n !}}(t-s)^{\frac{n}{2}}
$$

for all $s<t, n \geqslant 1$ and $1 \leqslant i_{1}, \cdots, i_{n} \leqslant d$.

Proof. By translation, it suffices to consider the case when $s=0$.

We first estimate the second moment of $\mathbb{B}_{0, u}^{n ; i_{1}, \cdots, i_{n}}$. Recall from (2.2) the shuffle product formula

$$
\mathbb{B}_{s, t}^{n ; i_{1}, \cdots, i_{n}} \cdot \mathbb{B}_{s, t}^{k ; i_{n+1}, \cdots, i_{n+k}}=\sum_{\sigma \in \mathcal{S}(n, k)} \mathbb{B}_{s, t}^{n+k ; i_{\sigma^{-1}(1)}, \cdots, i_{\sigma-1}(n+k)},
$$

where - is real number multiplication and $\mathcal{S}(n, k)$ denotes the set of all permutations on $\{1, \ldots, n+k\}$ such that

$$
\sigma(1)<\ldots<\sigma(n) ; \sigma(n+1)<\ldots<\sigma(n+k) .
$$


Applying this formula (3.1) and taking expectation, we have

$$
\mathbb{E}\left[\left|\mathbb{B}_{0, u}^{n ; i_{1}, \cdots, i_{n}}\right|^{2}\right]=\sum_{\sigma \in \mathcal{S}(n, n)} \mathbb{E}\left[\mathbb{B}_{0, u}^{2 n ; j^{-1}(1)}, \cdots, j_{\sigma^{-1}(2 n)}\right],
$$

where $\left(j_{1}, \cdots, j_{2 n}\right) \triangleq\left(i_{1}, \cdots, i_{n}, i_{1}, \cdots, i_{n}\right)$. Since $|\mathcal{S}(n, n)|=\frac{(2 n) !}{(n !)^{2}}$, we have

$$
\mathbb{E}\left[\left|\mathbb{B}_{0, u}^{n ; i_{1}, \cdots, i_{n}}\right|^{2}\right] \leq \frac{(2 n) !}{(n !)^{2}} \max _{\sigma \in \mathcal{S}(n, n)}\left|\mathbb{E}\left[\mathbb{B}_{0, u}^{2 n ; j^{-1}(1)}, \cdots, j_{\sigma^{-1}(2 n)}\right]\right|
$$

On the other hand, we know from [9] (see also Prop 4.10 in [17]) that

$$
\mathbb{E}\left[\mathbb{B}_{0, u}^{2 n}\right]=\frac{u^{n}}{n ! 2^{n}}\left(\sum_{i=1}^{d} \mathrm{e}_{i} \otimes \mathrm{e}_{i}\right)^{\otimes n}
$$

In particular, every coefficient of basis elements $\mathrm{e}_{k_{1}} \otimes \ldots \otimes \mathrm{e}_{k_{2 n}}$ in $\mathbb{E}\left[\mathbb{B}_{0, u}^{2 n}\right]$ is either zero or $\frac{u^{n}}{n ! 2^{n}}$. Therefore,

$$
\begin{aligned}
\mathbb{E}\left[\left|\mathbb{B}_{0, u}^{n ; i_{1}, \cdots, i_{n}}\right|^{2}\right] & \leqslant \frac{(2 n) !}{(n !)^{2}} \cdot \frac{u^{n}}{n ! 2^{n}} \\
& \leqslant \frac{\mathrm{e}(2 n)^{2 n+\frac{1}{2}} \mathrm{e}^{-2 n}}{2 \pi n^{2 n+1} \mathrm{e}^{-2 n}} \cdot \frac{u^{n}}{n ! 2^{n}} \quad \text { (by Stirling's approximation) } \\
& =\frac{\mathrm{e}}{\sqrt{2} \pi} \frac{2^{n}}{\sqrt{n} n !} u^{n} .
\end{aligned}
$$

Secondly, by the definition of iterated integral,

$$
\mathbb{B}_{0, u}^{n ; i_{1}, \cdots, i_{n}}=\int_{0}^{u} \mathbb{B}_{0, t}^{n-1 ; i_{1}, \cdots, i_{n-1}} \circ \mathrm{d} B_{t}^{i_{n}}
$$

and hence

$$
\begin{aligned}
d \mathbb{B}_{0, u}^{n ; i_{1}, \cdots, i_{n}} & =\mathbb{B}_{0, u}^{n-1 ; i_{1}, \cdots, i_{n-1}} \circ \mathrm{d} B_{u}^{i_{n}} \\
& =\mathbb{B}_{0, u}^{n-1 ; i_{1}, \cdots, i_{n-1}} \cdot \mathrm{d} B_{u}^{i_{n}}+\frac{1}{2} d \mathbb{B}_{0, u}^{n-1 ; i_{1}, \cdots, i_{n-1}} \cdot \mathrm{d} B_{u}^{i_{n}} \text { (Ito to Stratonovich) } \\
& =\mathbb{B}_{0, u}^{n-1 ; i_{1}, \cdots, i_{n-1}} \cdot \mathrm{d} B_{u}^{i_{n}}+\frac{1}{2}\left(\mathbb{B}_{0, u}^{n-2 ; i_{1}, \cdots, i_{n-2}} \circ d B_{u}^{i_{n-1}}\right) \cdot \mathrm{d} B_{u}^{i_{n}} \\
& =\mathbb{B}_{0, u}^{n-1 ; i_{1}, \cdots, i_{n-1}} \cdot \mathrm{d} B_{u}^{i_{n}}+\frac{1}{2} \delta_{i_{n-1}, i_{n}} \mathbb{B}_{0, u}^{n-2 ; i_{1}, \cdots, i_{n-2}} \mathrm{~d} u
\end{aligned}
$$


By integrating and taking supremum,

$$
\begin{aligned}
& \mathbb{E}\left[\sup _{0 \leqslant u \leqslant t}\left|\mathbb{B}_{0, u}^{n ; i_{1}, \cdots, i_{n}}\right|\right] \\
& \leqslant \mathbb{E}\left[\sup _{0 \leqslant u \leqslant t}\left|\int_{0}^{u} \mathbb{B}_{0, v}^{n-1 ; i_{1}, \cdots, i_{n-1}} \cdot \mathrm{d} B_{v}^{i_{n}}\right|\right]+\sup _{0 \leqslant u \leqslant t} \frac{1}{2} \int_{0}^{u} \mathbb{E}\left[\left|\mathbb{B}_{0, v}^{n-2 ; i_{1}, \cdots, i_{n-2}}\right|\right] \mathrm{d} v \\
& \leqslant \mathbb{E}\left[\sup _{0 \leqslant u \leqslant t}\left|\int_{0}^{u} \mathbb{B}_{0, v}^{n-1 ; i_{1}, \cdots, i_{n-1}} \cdot \mathrm{d} B_{v}^{i_{n}}\right|\right]+\frac{1}{2} \int_{0}^{t} \sqrt{\mathbb{E}\left[\left|\mathbb{B}_{0, v}^{n-2 ; i_{1}, \cdots, i_{n-2}}\right|^{2}\right]} \mathrm{d} v .
\end{aligned}
$$

It follows from (3.2) that

$$
\begin{aligned}
& \mathbb{E}\left[\sup _{0 \leqslant u \leqslant t}\left|\mathbb{B}_{0, u}^{n ; i_{1}, \cdots, i_{n}}\right|\right] \\
& \leqslant \mathbb{E}\left[\sup _{0 \leqslant u \leqslant t}\left|\int_{0}^{u} \mathbb{B}_{0, v}^{n-1 ; i_{1}, \cdots, i_{n-1}} \cdot \mathrm{d} B_{v}^{i_{n}}\right|\right]+\frac{1}{2} \int_{0}^{t}\left(\frac{\mathrm{e}}{\sqrt{2} \pi} \frac{2^{n-2}}{\sqrt{n-2}(n-2) !} v^{n-2}\right)^{\frac{1}{2}} \mathrm{~d} v \\
& =\mathbb{E}\left[\sup _{0 \leqslant u \leqslant t}\left|\int_{0}^{u} \mathbb{B}_{0, v}^{n-1 ; i_{1}, \cdots, i_{n-1}} \cdot \mathrm{d} B_{v}^{i_{n}}\right|\right]+\frac{1}{2}\left(\frac{\mathrm{e}}{\sqrt{2} \pi}\right)^{\frac{1}{2}} \frac{2^{\frac{n}{2}}}{(n-2)^{\frac{1}{4}} \sqrt{(n-2) ! n}} t^{\frac{n}{2}} \\
& \leqslant \mathbb{E}\left[\sup _{0 \leqslant u \leqslant t}\left|\int_{0}^{u} \mathbb{B}_{0, v}^{n-1 ; i_{1}, \cdots, i_{n-1}} \cdot \mathrm{d} B_{v}^{i_{n}}\right|\right]+\frac{1}{2}\left(\frac{\mathrm{e}}{\sqrt{2} \pi}\right)^{\frac{1}{2}} \frac{2^{\frac{n}{2}}}{(n-2)^{\frac{1}{4}} \sqrt{n !}} t^{\frac{n}{2}} .
\end{aligned}
$$

The first term can be estimated easily by using Doob's $L^{p}$-inequality:

$$
\begin{aligned}
& \mathbb{E}\left[\sup _{0 \leqslant u \leqslant t}\left|\int_{0}^{u} \mathbb{B}_{0, v}^{n-1 ; i_{1}, \cdots, i_{n-1}} \cdot \mathrm{d} B_{v}^{i_{n}}\right|\right] \leqslant\left\|\sup _{0 \leqslant u \leqslant t}\left|\int_{0}^{u} \mathbb{B}_{0, v}^{n-1 ; i_{1}, \cdots, i_{n-1}} \cdot \mathrm{d} B_{v}^{i_{n}}\right|\right\|_{2} \\
& \leqslant 2\left\|\int_{0}^{t} \mathbb{B}_{0, v}^{n-1 ; i_{1}, \cdots, i_{n-1}} \cdot \mathrm{d} B_{v}^{i_{n}}\right\|_{2} \\
& =2\left(\int_{0}^{t} \mathbb{E}\left[\left|\mathbb{B}_{0, v}^{n-1 ; i_{1}, \cdots, i_{n-1}}\right|^{2}\right] \mathrm{d} v\right)^{\frac{1}{2}} \\
& \leqslant 2\left(\int_{0}^{t} \frac{\mathrm{e}}{\sqrt{2} \pi} \frac{2^{n-1}}{\sqrt{n-1}(n-1) !} v^{n-1} \mathrm{~d} v\right)^{\frac{1}{2}} \\
& =\sqrt{2}\left(\frac{\mathrm{e}}{\sqrt{2} \pi}\right)^{\frac{1}{2}} \frac{2^{\frac{n}{2}}}{(n-2)^{\frac{1}{4}} \sqrt{n !}} t^{\frac{n}{2}} .
\end{aligned}
$$

Now the desired estimate follows immediately. 
Remark 3.1. Second moment estimate on iterated Stratonovich's integrals was studied by Ben Arous [1] through iterated Itô's integrals. Here the estimate (3.2) we obtained through the shuffle product formula and the Brownian expected signature is sharper in the exponential factor.

Now we are able to establish the following main upper estimate.

Proposition 3.1. Suppose that the tensor products $\left(\mathbb{R}^{d}\right)^{\otimes n}$ are equipped with given admissible norms, under which each element of the standard basis $\left\{\mathrm{e}_{1}, \cdots, \mathrm{e}_{d}\right\}$ of $\mathbb{R}^{d}$ has norm one. Then for each $s<t$, with probability one, we have

$\max \left\{\limsup _{n \rightarrow \infty}\left(\left(\frac{n}{2}\right) ! \sup _{s \leqslant u \leqslant t}\left\|\mathbb{B}_{s, u}^{n}\right\|\right)^{\frac{2}{n}}, \limsup _{n \rightarrow \infty}\left(\left(\frac{n}{2}\right) ! \sup _{s \leqslant u \leqslant t}\left\|\mathbb{B}_{u, t}^{n}\right\|\right)^{\frac{2}{n}}\right\} \leqslant d^{2}(t-s)$.

Proof. Since the tensor norms are admissible, for each multi-index,

$$
\left\|\mathrm{e}_{i_{1}} \otimes \cdots \otimes \mathrm{e}_{i_{n}}\right\| \leq\left\|\mathrm{e}_{i_{1}}\right\|\left\|\mathrm{e}_{i_{2}}\right\| \ldots\left\|\mathrm{e}_{i_{n}}\right\|=1 .
$$

This together with the triangle inequality implies that

$$
\begin{aligned}
\left\|\mathbb{B}_{s, u}^{n}\right\| & =\left\|\sum_{i_{1}, \cdots, i_{n}=1}^{d} \mathbb{B}_{s, u}^{n ; i_{1}, \cdots, i_{n}} \mathrm{e}_{i_{1}} \otimes \cdots \otimes \mathrm{e}_{i_{n}}\right\| \\
& \leqslant \sum_{i_{1}, \cdots, i_{n}=1}^{d}\left|\mathbb{B}_{s, u}^{n ; i_{1}, \cdots, i_{n}}\right|,
\end{aligned}
$$

and thus

$$
\mathbb{E}\left[\sup _{s \leqslant u \leqslant t}\left\|\mathbb{B}_{s, t}^{n}\right\|\right] \leqslant \sum_{i_{1}, \cdots, i_{n}=1}^{d} \mathbb{E}\left[\sup _{s \leqslant u \leqslant t}\left|\mathbb{B}_{s, u}^{n ; i_{1}, \cdots, i_{n}}\right|\right] .
$$

The summand on the right hand side of (3.6) can be bounded by Lemma 3.1, and we arrive at

$$
\mathbb{E}\left[\sup _{s \leqslant u \leqslant t}\left\|\mathbb{B}_{s, u}^{n}\right\|\right] \leqslant d^{n} \cdot \frac{C 2^{\frac{n}{2}}}{(n-2)^{\frac{1}{4}} \sqrt{n !}}(t-s)^{\frac{n}{2}},
$$

where

$$
C \triangleq\left(\frac{1}{2}+\sqrt{2}\right)\left(\frac{\mathrm{e}}{\sqrt{2} \pi}\right)^{\frac{1}{2}} \text {. }
$$

Now for each $r>(t-s)$, we have

$$
\mathbb{P}\left(\sup _{s \leqslant u \leqslant t}\left\|\mathbb{B}_{s, u}^{n}\right\|>\frac{C d^{n} 2^{\frac{n}{2}}}{(n-2)^{\frac{1}{4}} \sqrt{n !}} r^{\frac{n}{2}}\right) \leqslant\left(\frac{t-s}{r}\right)^{\frac{n}{2}} .
$$


By the Borel-Cantelli lemma, with probability one (with null set depending on $s$ and $t$ ),

$$
\sup _{s \leqslant u \leqslant t}\left\|\mathbb{B}_{s, u}^{n}\right\| \leqslant \frac{C d^{n} 2^{\frac{n}{2}}}{(n-2)^{\frac{1}{4}} \sqrt{n !}} r^{\frac{n}{2}}
$$

for all sufficiently large $n$. It follows from Stirling's approximation that with probability one,

$$
\limsup _{n \rightarrow \infty}\left(\left(\frac{n}{2}\right) ! \sup _{s \leqslant u \leqslant t}\left\|\mathbb{B}_{s, u}^{n}\right\|\right)^{\frac{2}{n}} \leqslant \lim _{n \rightarrow \infty}\left(\left(\frac{n}{2}\right) ! \frac{C d^{n} 2^{\frac{n}{2}}}{(n-2)^{\frac{1}{4}} \sqrt{n !}} r^{\frac{n}{2}}\right)^{\frac{2}{n}}=d^{2} r .
$$

By taking a rational sequence $r \downarrow(t-s)$, we conclude that with probability one (with null set depending on $t$ and $s$ ),

$$
\limsup _{n \rightarrow \infty}\left(\left(\frac{n}{2}\right) ! \sup _{s \leqslant u \leqslant t}\left\|\mathbb{B}_{s, u}^{n}\right\|\right)^{\frac{2}{n}} \leqslant d^{2}(t-s) .
$$

Next we will bound $\sup _{v \leq u \leq t}\left\|\mathbb{B}_{u, t}^{n}\right\|$ using the reversability of Brownian motion. For the estimate involving $\overline{\mathbb{B}}_{u, t}^{n}$, observe that

$$
\begin{aligned}
\mathbb{B}_{u, t}^{n} & =\int_{u<v_{1}<\cdots<v_{n}<t} \mathrm{~d} B_{v_{1}} \otimes \cdots \otimes \mathrm{d} B_{v_{n}} \\
& =\int_{0<r_{n}<\cdots<r_{1}<t-u} \mathrm{~d} B_{t-r_{1}} \otimes \cdots \otimes \mathrm{d} B_{t-r_{n}} \\
& =\mathcal{P}^{\tau}\left(\int_{0<r_{1}<\cdots<r_{n}<t-u} \mathrm{~d} W_{r_{1}} \otimes \cdots \otimes \mathrm{d} W_{r_{n}}\right),
\end{aligned}
$$

where $W_{r} \triangleq B_{t-r}-B_{t}(0 \leqslant r \leqslant t-u)$ is again a Brownian motion and $\mathcal{P}^{\tau}$ is the linear transformation on $\left(\mathbb{R}^{d}\right)^{\otimes n}$ determined by $\xi_{1} \otimes \cdots \otimes \xi_{n} \mapsto \xi_{n} \otimes \cdots \otimes \xi_{1}$. It follows that

$$
\left\|\mathbb{B}_{u, t}^{n}\right\|=\left\|\mathbb{W}_{0, t-u}^{n}\right\|
$$

Therefore,

$$
\sup _{s \leqslant u \leqslant t}\left\|\mathbb{B}_{u, t}^{n}\right\|=\sup _{0 \leqslant v \leqslant t-s}\left\|\mathbb{W}_{0, v}^{n}\right\| .
$$

Therefore, what we have proven before shows that

$$
\begin{aligned}
\limsup _{n \rightarrow \infty}\left(\left(\frac{n}{2}\right) ! \sup _{s \leqslant u \leqslant t}\left\|\mathbb{B}_{u, t}^{n}\right\|\right)^{\frac{2}{n}} & =\limsup _{\substack{n \rightarrow \infty\\
}}\left(\left(\frac{n}{2}\right) ! \sup _{0 \leqslant v \leqslant t-s}\left\|\mathbb{W}_{0, v}^{n}\right\|\right)^{\frac{2}{n}} \\
& \leqslant d^{2}(t-s)
\end{aligned}
$$

for almost surely. 
Recall that $\widetilde{L}_{s, t}$ is defined by

$$
\widetilde{L}_{s, t} \triangleq \limsup _{n \rightarrow \infty}\left(\left(\frac{n}{2}\right) !\left\|\int_{s<t_{1}<\cdots<t_{n}<t} \operatorname{od} B_{t_{1}} \otimes \cdots \otimes \circ \mathrm{od} B_{t_{n}}\right\|\right)^{\frac{2}{n}}
$$

under given admissible tensor norms. It is immediate from Proposition 3.1 that $\widetilde{L}_{s, t} \leqslant d^{2}(t-s)$ for almost surely.

Now we are going to show that $\widetilde{L}_{s, t}$ is almost surely a deterministic constant.

Recall that $g \in T\left(\left(\mathbb{R}^{d}\right)\right)$ is a group-like element if and only if $g=\left(1, g^{1}, g^{2}, \cdots\right)$ satisfies

$$
g^{n} \otimes g^{k}=\sum_{\sigma \in \mathcal{S}(n, k)} \mathcal{P}^{\sigma}\left(g^{n+k}\right)
$$

where, as mentioned, $\mathcal{P}^{\sigma}$ is the unique linear map on $\left(\mathbb{R}^{d}\right)^{\otimes(n+k)}$ such that

$$
\mathcal{P}^{\sigma}\left(v_{1} \otimes \ldots \otimes v_{n+k}\right)=v_{\sigma(1)} \otimes \ldots \otimes v_{\sigma(n+k)}
$$

and $\mathcal{S}(n, k)$ denotes the set of permutations on $n+k$ elements such that

$$
\sigma(1)<\sigma(2)<\ldots<\sigma(n), \sigma(n+1)<\ldots<\sigma(n+k) .
$$

This is in fact equivalent to the shuffle product formula (3.1) mentioned earlier. In particular, the signature of a geometric rough path is always a group-like element.

Lemma 3.2. Let $g=\left(1, g^{1}, g^{2}, \cdots\right)$ be a non-trivial group-like element in the tensor algebra $T\left(\left(\mathbb{R}^{d}\right)\right)$, where the tensor products are equipped with given admissible norms. Then $g$ has infinitely many non-zero components.

Proof. Suppose that $g^{k} \neq 0$ for some $k \geqslant 1$. According to the shuffle product formula, for each $n \geqslant 1$,

$$
\left(g^{k}\right)^{\otimes n}=\sum_{\sigma \in \mathcal{S}(k, \cdots, k)} \mathcal{P}^{\sigma}\left(g^{n k}\right)
$$

with $\mathcal{S}(k, \ldots, k)$ denoting the set of permutations on $n k$ elements such that

$$
\begin{aligned}
\sigma(1) & <\ldots<\sigma(k) ; \\
\sigma(k+1) & <\ldots<\sigma(2 k) ; \\
\vdots & \\
\sigma((n-1) k+1) & <\ldots<\sigma(n k) .
\end{aligned}
$$


Since the tensor norms are admissible, we have

$$
\begin{aligned}
\left\|g^{k}\right\|^{n} & \leqslant \sum_{\sigma \in \mathcal{S}(k, \cdots, k)}\left\|\mathcal{P}^{\sigma}\left(g^{n k}\right)\right\| \\
& =\frac{(n k) !}{(k !)^{n}}\left\|g^{n k}\right\| .
\end{aligned}
$$

In particular, $g^{n k} \neq 0$ for all $n$.

Lemma 3.3. Given $\alpha>0$, there exists a constant $C>0$, such that

$$
\frac{\left(\frac{n}{p}\right) !}{\left(\frac{n-\alpha}{p}\right) !} \leqslant C n^{\frac{\alpha}{p}}, \quad \forall n>2 \alpha, p \geqslant 1 .
$$

Proof. According to Stirling's approximation, there exist constants $C_{1}, C_{2}>0$, such that

$$
C_{1} \lambda^{\lambda+\frac{1}{2}} \mathrm{e}^{-\lambda} \leqslant \lambda ! \leqslant C_{2} \lambda^{\lambda+\frac{1}{2}} \mathrm{e}^{-\lambda}, \quad \forall \lambda>0
$$

Therefore,

$$
\begin{aligned}
\frac{\left(\frac{n}{p}\right) !}{\left(\frac{n-\alpha}{p}\right) !} & \leqslant \frac{C_{2}\left(\frac{n}{p}\right)^{\frac{n}{p}+\frac{1}{2}} \mathrm{e}^{-\frac{n}{p}}}{C_{1}\left(\frac{n-\alpha}{p}\right)^{\frac{n-\alpha}{p}+\frac{1}{2}} \mathrm{e}^{-\frac{n-\alpha}{p}}} \\
& =\frac{C_{2}}{C_{1}(p \mathrm{e})^{\frac{\alpha}{p}}}\left(1+\frac{\alpha}{n-\alpha}\right)^{\frac{n-\alpha}{p}+\frac{1}{2}} n^{\frac{\alpha}{p}} \\
& \leqslant \frac{\sqrt{2} C_{2} \mathrm{e}^{\alpha}}{C_{1}} n^{\frac{\alpha}{p}} .
\end{aligned}
$$

Choosing $C \triangleq \sqrt{2} C_{2} \mathrm{e}^{\alpha} / C_{1}$ suffices.

The following deterministic sub-additivity property is essential for us.

Proposition 3.2. (Subadditivity estimate) Suppose that $\mathbf{X}$ is a rough path, where the tensor products are equipped with given admissible norms. Let $\mathbb{X}_{s, t}^{n}$ be the degree- $n$ iterated integrals of $\mathbf{X}$ on the interval $[s, t]$, as defined by Theorem 2.1. Let $p \geqslant 1$ be a given constant. Define

$$
\widetilde{l}_{s, t} \triangleq \limsup _{n \rightarrow \infty}\left\|\left(\frac{n}{p}\right) ! \mathbb{X}_{s, t}^{n}\right\|^{\frac{p}{n}}, \quad s \leqslant t
$$


Then $(s, t) \mapsto \widetilde{l}_{s, t}$ is sub-additive, i.e.

$$
\tilde{l}_{s, t} \leqslant \tilde{l}_{s, u}+\tilde{l}_{u, t}
$$

for $s \leqslant u \leqslant t$

Proof. We may assume that $\widetilde{l}_{s, u}, \widetilde{l}_{u, t}$ are both finite. Moreover, we may also assume that both of $\mathbb{X}_{s, u}$ and $\mathbb{X}_{u, t}$ are non-trivial, otherwise the desired inequality is trivial due to the multiplicative property (2.4). From Lemma 3.2, $\mathbb{X}_{s, u}$ and $\mathbb{X}_{u, t}$ have infinitely many non-zero components.

Given integers $\alpha>2 p$ and $n>2 \alpha$, according to the multiplicative property (2.4), we have

$$
\begin{aligned}
\left\|\mathbb{X}_{s, t}^{n}\right\|= & \left\|\sum_{k=0}^{n} \mathbb{X}_{s, u}^{k} \otimes \mathbb{X}_{u, t}^{n-k}\right\| \\
\leqslant & \sum_{k=0}^{\alpha-1}\left\|\mathbb{X}_{s, u}^{n-k}\right\| \cdot\left\|\mathbb{X}_{u, t}^{k}\right\|+\sum_{k=n-\alpha+1}^{n}\left\|\mathbb{X}_{s, u}^{n-k}\right\| \cdot\left\|\mathbb{X}_{u, t}^{k}\right\| \\
& +\sum_{k=\alpha}^{n-\alpha}\left\|\mathbb{X}_{s, u}^{n-k}\right\| \cdot\left\|\mathbb{X}_{u, t}^{k}\right\|
\end{aligned}
$$

Define $(s, t) \mapsto \widetilde{l}_{s, t}^{\alpha} \triangleq \sup _{k \geqslant \alpha}\left\|(k / p) ! \mathbb{X}_{s, t}^{k}\right\|^{p / k}$. It follows that

$$
\begin{aligned}
\left\|\mathbb{X}_{s, t}^{n}\right\| \leqslant & \sum_{k=0}^{\alpha-1} \frac{\left(\widetilde{l}_{s, u}^{\alpha}\right)^{\frac{n-k}{p}}}{\left(\frac{n-k}{p}\right) !} \cdot\left\|\mathbb{X}_{u, t}^{k}\right\|+\sum_{k=n-\alpha+1}^{n} \frac{\left(\widetilde{l}_{u, t}^{\alpha}\right)^{\frac{k}{p}}}{\left(\frac{k}{p}\right) !} \cdot\left\|\mathbb{X}_{s, u}^{n-k}\right\| \\
& +\sum_{k=\alpha}^{n-\alpha} \frac{\left(\widetilde{l}_{s, u}^{\alpha}\right)^{\frac{n-k}{p}}}{\left(\frac{n-k}{p}\right) !} \cdot \frac{\left(\widetilde{l}_{u, t}^{\alpha}\right)^{\frac{k}{p}}}{\left(\frac{k}{p}\right) !} \\
\leqslant & \sum_{k=0}^{\alpha-1} \frac{\left(\widetilde{l}_{s, u}^{\alpha}\right)^{\frac{n-k}{p}}}{\left(\frac{n-k}{p}\right) !} \cdot\left\|\mathbb{X}_{u, t}^{k}\right\|+\sum_{k=n-\alpha+1}^{n} \frac{\left(\widetilde{l}_{u, t}^{\alpha}\right)^{\frac{k}{p}}}{\left(\frac{k}{p}\right) !} \cdot\left\|\mathbb{X}_{s, u}^{n-k}\right\| \\
& +p \frac{\left(\widetilde{l}_{s, u}^{\alpha}+\widetilde{l}_{u, t}^{\alpha}\right)^{\frac{n}{p}}}{\left(\frac{n}{p}\right) !},
\end{aligned}
$$


where in the final inequality we have used the neo-classical inequality (c.f. [13]), which states that

$$
\sum_{i=0}^{N} \frac{a^{\frac{i}{p}} b^{\frac{N-i}{p}}}{\left(\frac{i}{p}\right) !\left(\frac{N-i}{p}\right) !} \leqslant p \frac{(a+b)^{\frac{N}{p}}}{\left(\frac{N}{p}\right) !}, \quad \forall a, b \geqslant 0, p \geqslant 1, N \in \mathbb{N} .
$$

Multiplying through by $\left(\frac{n}{p}\right)$ ! and using Lemma 3.3 which states that $\left(\frac{n}{p}\right)$ ! $\leq$ $C n^{\frac{\alpha}{p}}\left(\frac{n-\alpha}{p}\right) !$

$$
\begin{aligned}
& \left(\frac{n}{p}\right) !\left\|\mathbb{X}_{s, t}^{n}\right\| \\
& \leqslant \frac{\left(\frac{n}{p}\right) !}{\left(\frac{n-\alpha}{p}\right) !}\left(\sum_{k=0}^{\alpha-1}\left(\widetilde{l}_{s, u}^{\alpha}\right)^{\frac{n-k}{p}} \cdot\left\|\mathbb{X}_{u, t}^{k}\right\|+\sum_{k=n-\alpha+1}^{n}\left(\widetilde{l}_{u, t}^{\alpha}\right)^{\frac{k}{p}} \cdot\left\|\mathbb{X}_{s, u}\right\|^{n-k}\right) \\
& \quad+p\left(\widetilde{l}_{s, u}^{\alpha}+\widetilde{l}_{u, t}^{\alpha}\right)^{\frac{n}{p}} \\
& \leqslant C n^{\frac{\alpha}{p}}\left(\sum_{k=0}^{\alpha-1}\left(\widetilde{l}_{s, u}^{\alpha}\right)^{\frac{n-k}{p}} \cdot\left\|\mathbb{X}_{u, t}^{k}\right\|+\sum_{k=n-\alpha+1}^{n}\left(\widetilde{l}_{u, t}^{\alpha}\right)^{\frac{k}{p}} \cdot\left\|\mathbb{X}_{s, u}\right\|^{n-k}\right) \\
& \quad+p\left(\widetilde{l}_{s, u}^{\alpha}+\widetilde{l}_{u, t}^{\alpha}\right)^{\frac{n}{p}} .
\end{aligned}
$$

Therefore,

$$
\widetilde{l}_{s, t}=\limsup _{n \rightarrow \infty}\left(\left(\frac{n}{p}\right) !\left\|\mathbb{X}_{s, t}^{n}\right\|\right)^{\frac{p}{n}} \leqslant \widetilde{l}_{s, u}^{\alpha}+\widetilde{l}_{u, t}^{\alpha},
$$

where we have used the simple fact that

$$
\lim _{n \rightarrow \infty}\left(\left(\lambda a^{\frac{n}{p}}+\mu b^{\frac{n}{p}}\right) n^{\nu}+(a+b)^{\frac{n}{p}}\right)^{\frac{p}{n}}=a+b
$$

for any $\lambda, \mu, \nu, a, b, p>0$ (note that, as discussed at the beginning of the proof, $\left.\widetilde{l}_{s, u}^{\alpha}, \widetilde{l}_{u, t}^{\alpha}>0\right)$.

Now the result follows from taking $\alpha \rightarrow \infty$ in (3.8).

Remark 3.2. Typically if $\mathbf{X}$ has finite $p$-variation, then from Lyons' extension theorem we know that $\widetilde{l}_{s, t}$ is finite.

Remark 3.3. This is a side remark related to whether the reverse inequality in Lemma 3.2 holds. Note that Lemma 3.2 holds for all geometric rough paths $\mathbf{X}$ 
regardless of whether $\mathbf{X}$ has a tree-like piece (a tree-like piece is a loop in an $\mathbb{R}$ tree, see [3] or [11] for the precise definition). On the other hand, a superadditive estimate of the form

$$
\widetilde{l}_{s, t} \geqslant \widetilde{l}_{s, u}+\widetilde{l}_{u, t}
$$

if true at all, can at best only hold for tree-reduced paths, as inserting tree-like pieces could make the right hand side of (3.9) arbitrarily big while leaving the left hand side unchanged.

Theorem 3.1. Let the tensor products over $\mathbb{R}^{d}$ be equipped with given admissible norms, under which each element of the standard basis $\left\{\mathrm{e}_{1}, \cdots, \mathrm{e}_{d}\right\}$ of $\mathbb{R}^{d}$ has norm one. Then for each $s<t, \widetilde{L}_{s, t}$ is almost surely a deterministic constant which is bounded above by $d^{2}(t-s)$.

Proof. For $m \geqslant 1$, consider the dyadic partition

$$
t_{i}^{m} \triangleq s+\frac{i}{2^{m}}(t-s), \quad i=0, \cdots, 2^{m} .
$$

According to the subadditivity estimate, Proposition 3.2, we know that pathwisely

$$
\widetilde{L}_{s, t} \leqslant \sum_{i=1}^{2^{m}} \widetilde{L}_{t_{i-1}^{m}, t_{i}^{m}}=2^{-m} \sum_{i=1}^{2^{m}} 2^{m} \widetilde{L}_{t_{i-1}^{m}, t_{i}^{m}} .
$$

On the one hand, by the Brownian scaling, for each $i, 2^{m} \widetilde{L}_{t_{i-1}^{m}, t_{i}^{m}}$ has the same distribution as $\widetilde{L}_{s, t}$. In particular, by Proposition 3.1, it is bounded above by $d^{2}(t-s)$ almost surely. On the other hand, the family $\left\{2^{m} \widetilde{L}_{t_{i-1}^{m}, t_{i}^{m}}: 1 \leqslant i \leqslant 2^{m}\right\}$ are independent. According to the weak law of large numbers, we conclude that

$$
2^{-m} \sum_{i=1}^{2^{m}} 2^{m} \widetilde{L}_{t_{i-1}^{m}, t_{i}^{m}} \rightarrow \mathbb{E}\left[\widetilde{L}_{s, t}\right]
$$

in probability. By taking an almost surely convergent subsequence, we obtain that

$$
\widetilde{L}_{s, t} \leqslant \mathbb{E}\left[\widetilde{L}_{s, t}\right]
$$

almost surely. This certainly implies that $\widetilde{L}_{s, t}=\mathbb{E}\left[\widetilde{L}_{s, t}\right]$ almost surely.

Remark 3.4. Although the fact of $\widetilde{L}_{s, t}$ being a deterministic constant is a result of independent increments for Brownian motion, it is not clear that any simple type of 0-1 law argument could apply. 
Corollary 3.1. Under the assumption of Theorem 3.1, there exists a constant $\kappa_{d}$ depending on $d$, such that for each pair of $s<t$, with probability one we have $\widetilde{L}_{s, t}=\kappa_{d}(t-s)$.

Proof. The result follows immediately from Theorem 3.1 and Brownian scaling.

Remark 3.5. We should emphasize that the constant $\kappa_{d}$ depends on the choice of given admissible norms on the tensor products.

We can further show that the $\mathbb{P}$-null set arising from Corollary 3.1 associated with each pair of $s<t$ can be chosen to be universal. This point will be very useful for applications to the level of the Brownian rough path (c.f. Section 6 below).

Proposition 3.3. With probability one, we have

$$
\widetilde{L}_{s, t}=\kappa_{d}(t-s) \text { for all } s<t .
$$

Proof. According to Proposition 3.1 and Corollary 3.1, there exists a $\mathbb{P}$-null set $\mathcal{N}$, such that for all $\omega \notin \mathcal{N}$, we have

$$
\max \left\{L_{r_{1}, r_{2}}^{\prime}(\omega), L_{r_{1}, r_{2}}^{\prime \prime}(\omega)\right\} \leqslant d^{2}\left(r_{2}-r_{1}\right)
$$

and

$$
\widetilde{L}_{r_{1}, r_{2}}(\omega)=\kappa_{d}\left(r_{2}-r_{1}\right)
$$

for all $r_{1}, r_{2} \in \mathbb{Q}$ with $r_{1}<r_{2}$, where

$$
\begin{aligned}
& L_{r_{1}, r_{2}}^{\prime} \triangleq \limsup _{n \rightarrow \infty}\left(\left(\frac{n}{2}\right) ! \sup _{r_{1} \leqslant u \leqslant r_{2}}\left\|\mathbb{B}_{r_{1}, u}^{n}\right\|\right)^{\frac{2}{n}}, \\
& L_{r_{1}, r_{2}}^{\prime \prime} \triangleq \limsup _{n \rightarrow \infty}\left(\left(\frac{n}{2}\right) ! \sup _{r_{1} \leqslant u \leqslant r_{2}}\left\|\mathbb{B}_{u, r_{2}}^{n}\right\|\right)^{\frac{2}{n}} .
\end{aligned}
$$

Now fix $\omega \notin \mathcal{N}$ and let $s<r$ with $r \in \mathbb{Q}$. For arbitrary $r_{1}, r_{2} \in \mathbb{Q}$ with $r_{1}<s<r_{2}$, we know that

$$
\begin{aligned}
\kappa_{d}\left(r-r_{1}\right) & =\widetilde{L}_{r_{1}, r}(\omega) \\
& \leqslant \widetilde{L}_{r_{1}, s}(\omega)+\widetilde{L}_{s, r}(\omega) \text { (by subadditivity estimate, Prop. 3.2) } \\
& \leqslant L_{r_{1}, r_{2}}^{\prime}(\omega)+\widetilde{L}_{s, r}(\omega) \\
& \leqslant d^{2}\left(r_{2}-r_{1}\right)+\widetilde{L}_{s, r}(\omega) \text { (by Prop. 3) }
\end{aligned}
$$


By letting $r_{1} \uparrow s$ and $r_{2} \downarrow s$ along rational times, we obtain that

$$
\widetilde{L}_{s, r}(\omega) \geqslant \kappa_{d}(r-s) .
$$

Similarly, from

$$
\begin{aligned}
\widetilde{L}_{s, r}(\omega) & \leqslant \widetilde{L}_{s, r_{2}}(\omega)+\widetilde{L}_{r_{2}, r}(\omega) \text { (by subadditivity estimate, Prop. 3.2) } \\
& \leqslant L_{r_{1}, r_{2}}^{\prime \prime}(\omega)+\widetilde{L}_{r_{2}, r}(\omega) \\
& \leqslant d^{2}\left(r_{2}-r_{1}\right)+\kappa_{d}\left(r-r_{2}\right), \text { (by Prop. 3) }
\end{aligned}
$$

we conclude that

$$
\widetilde{L}_{s, r}(\omega) \leqslant \kappa_{d}(r-s) .
$$

Therefore,

$$
\widetilde{L}_{s, r}(\omega)=\kappa_{d}(r-s) .
$$

By repeating the same argument to the parameter $r$, we conclude that for all $s<t$,

$$
\widetilde{L}_{s, t}(\omega)=\kappa_{d}(t-s) .
$$

\section{The second part of the main result: the lower estimate}

For given admissible tensor norms, from the last section we know that with probability one,

$$
\widetilde{L}_{s, t}=\kappa_{d}(t-s),
$$

where $\kappa_{d}$ is a deterministic constant depending only on the dimension $d$ of Brownian motion, which is bounded above by $d^{2}$. It is not even clear that $\kappa_{d}$ should be strictly positive. In this section, we are going to establish a lower estimate of $\kappa_{d}$ under the projective tensor norm by applying the technique of hyperbolic development which was introduced by Hambly and Lyons' paper [12]. In the next section, we shall see that the positivity of $\kappa_{d}$ reflects certain non-degeneracy properties of the Brownian rough path. 


\subsection{The hyperbolic development of a regular path}

Before studying the Brownian signature, let us first summarize the fundamental idea of hyperbolic development in the deterministic context for regular paths. We present proofs of a few results which seems not appearing in the literature. For an expository review on hyperbolic geometry, we refer the reader to the wonderful survey [5].

Let $\mathbb{H}^{d}(d \geqslant 2)$ be the complete, connected and simply-connected $d$-dimensional Riemannian manifold with constant sectional curvature -1. For computational convenience, we choose the hyperboloid model. In particular, $\mathbb{H}^{d}$ is defined to be the submanifold $\left\{x \in \mathbb{R}^{d+1}: x * x=-1, x^{d+1}>0\right\}$, where $*$ is the Minkowski metric on $\mathbb{R}^{d+1}$ given by

$$
x * y \triangleq \sum_{i=1}^{d} x^{i} y^{i}-x^{d+1} y^{d+1} .
$$

The Minkowski metric induces a Riemannian metric on $\mathbb{H}^{d}$ which gives it the desired hyperbolic structure. For $x, y \in \mathbb{H}^{d}$, one can show that

$$
\cosh \rho(x, y)=-x * y,
$$

where $\rho(x, y)$ is the hyperbolic distance between $x$ and $y$.

It is known that the isometry group $\mathrm{SO}(d, 1)$ of $\mathbb{H}^{d}$ is the space of $(d+1) \times$ $(d+1)$-invertible matrices $\Gamma$ such that $\Gamma^{-1}=J \Gamma^{*} J$ and $\Gamma_{d+1}^{d+1}>0$, where $J \triangleq$ $\operatorname{diag}(1, \cdots, 1,-1)$. The Lie algebra $\operatorname{so}(d, 1)$ of $\mathrm{SO}(d, 1)$ is the space of $(d+1) \times$ $(d+1)$-matrices $A$ of the form

$$
A=\left(\begin{array}{cc}
A_{0} & b \\
b^{*} & 0
\end{array}\right)
$$

where $A_{0}$ is an antisymmetric $d \times d$-matrix and $b \in \mathbb{R}^{d}$.

Define a linear map $F: \mathbb{R}^{d} \rightarrow \operatorname{so}(d, 1)$ by

$$
F(x) \triangleq\left(\begin{array}{cccc}
0 & \cdots & 0 & x^{1} \\
\vdots & \ddots & \vdots & \vdots \\
0 & \cdots & 0 & x^{d} \\
x^{1} & \cdots & x^{d} & 0
\end{array}\right), \quad x=\left(x^{1}, \cdots, x^{d}\right) \in \mathbb{R}^{d} .
$$

Given a continuous path $\gamma:[0,1] \rightarrow \mathbb{R}^{d}$ with bounded variation, consider the linear ordinary differential equation

$$
\left\{\begin{array}{l}
d \Gamma_{t}=\Gamma_{t} F\left(\mathrm{~d} \gamma_{t}\right), \quad t \in[0,1], \\
\Gamma_{0}=\mathrm{I}_{d+1} .
\end{array}\right.
$$


The solution $\Gamma_{t}$ defines a continuous path with bounded variation in the isometry group $\mathrm{SO}(d, 1)$. Explicitly, by Picard's iteration, we see that

$$
\Gamma_{t}=\sum_{n=0}^{\infty} \int_{0<t_{1}<\cdots<t_{n}<t} F\left(\mathrm{~d} \gamma_{t_{1}}\right) \cdots F\left(\mathrm{~d} \gamma_{t_{n}}\right)=\sum_{n=0}^{\infty} F^{\otimes n}\left(g_{n}(t)\right),
$$

where we recall that $g_{n}(t) \triangleq \int_{0<t_{1}<\ldots<t_{n}<t} \mathrm{~d} \gamma_{t_{1}} \otimes \ldots \otimes \mathrm{d} \gamma_{t_{n}}$. Define $X_{t} \triangleq \Gamma_{t} o$, where $o=(0, \cdots, 0,1)^{*} \in \mathbb{H}^{d}$.

Definition 4.1. $\Gamma_{t}$ is called the Cartan development of $\gamma_{t}$ onto $\mathrm{SO}(d, 1) . X_{t}$ is called the hyperbolic development of $\gamma_{t}$ onto $\mathbb{H}^{d}$.

The reason of expecting a lower estimate of $\kappa_{d}$ in our Brownian setting from the hyperbolic development is quite related to the philosophy in the setting of bounded variation paths. To be precise, define

$$
\widetilde{l} \triangleq \sup _{n \geqslant 1}\left(n !\left\|g_{n}\right\|_{\text {proj }}\right)^{\frac{1}{n}} \leqslant\|\gamma\|_{1-\mathrm{var}}
$$

where $g_{n} \triangleq \int_{0<t_{1}<\cdots<t_{n}<1} \mathrm{~d} \gamma_{t_{1}} \otimes \cdots \otimes \mathrm{d} \gamma_{t_{n}}$ is the $n$-th component of the signature of $\gamma$, and $\|\cdot\|_{\text {proj }}$ is the projective tensor norm induced by the Euclidean norm on $\mathbb{R}^{d}$.

Now suppose that $\gamma$ is tree-reduced. There are essentially two cases in which the length conjecture $\widetilde{l}=\|\gamma\|_{1-\text { var }}$ is known to be true: piecewise linear paths or $C^{1}$-paths in constant speed parametrization ([12, 18]).

The fundamental reason that the hyperbolic development yields the lower bound $\tilde{l} \geqslant\|\gamma\|_{1-\text { var }}$ is hidden in the following two key facts.

Fact 1. The hyperbolic development is length preserving. Moreover, if $\gamma_{t}$ is piecewise linear, then its hyperbolic development $X_{t}$ is piecewise geodesic with the same intersection angles as those of $\gamma_{t}$.

Proof. We first show that the Cartan development is length preserving.

If $\gamma_{t}$ is smooth, then the equation for $\Gamma_{t}$ becomes

$$
\dot{\Gamma}_{t}=\Gamma_{t} F\left(\dot{\gamma}_{t}\right)
$$

and thus

$$
\dot{X}_{t}=\dot{\Gamma}_{t} O=\Gamma_{t}\left(\begin{array}{c}
\dot{\gamma}_{t} \\
0
\end{array}\right)
$$


Since $\Gamma_{t}$ is an isometry of $\mathbb{H}^{d}$, by identifying $T_{o} \mathbb{H}^{d} \cong \mathbb{R}^{d}$, we conclude that

$$
\left\|\dot{X}_{t}\right\|_{*}=\left\|\left(\begin{array}{c}
\dot{\gamma}_{t} \\
0
\end{array}\right)\right\|_{*}=\left\|\dot{\gamma}_{t}\right\|_{\text {Euclidean }}
$$

where we define

$$
\left\|\left(\begin{array}{c}
x_{1} \\
\vdots \\
x_{d+1}
\end{array}\right)\right\|_{*} \triangleq \sqrt{\left(\begin{array}{c}
x_{1} \\
\vdots \\
x_{d+1}
\end{array}\right) *\left(\begin{array}{c}
x_{1} \\
\vdots \\
x_{d+1}
\end{array}\right)}=\sqrt{\sum_{i=1}^{d} x_{i}^{2}-x_{d+1}^{2}} .
$$

It follows that the hyperbolic length of $X_{t}$ is the same as the Euclidean length of $\gamma_{t}$. The general bounded variation case can be proved by smooth approximation.

Next we show that the Cartan development of a piecewise linear path is a piecewise geodesic with the same intersection angles.

If $\gamma_{t}=t v$ is a linear path, it can be shown using (4.2) that

$$
X_{1}^{d+1}=\left(\Gamma_{1} o\right)^{d+1}=\sum_{n=0}^{\infty} \frac{\|v\|_{\text {Euclidean }}^{2 n}}{(2 n) !}=\cosh \|v\|_{\text {Euclidean }} .
$$

(As this equality is given for motivation only, we will not give a proof.) From the identity (4.1), we know that

$$
\cosh \rho\left(X_{1}, o\right)=-X_{1} * o=X_{1}^{d+1}=\cosh \|v\|_{\text {Euclidean }},
$$

which implies that

$$
\rho\left(X_{1}, o\right)=\|v\|_{\text {Euclidean }}=\|\gamma\|_{1-\text { var }} .
$$

Therefore, $X$ is a geodesic in $\mathbb{H}_{d}$.

Now suppose that $\gamma_{t}$ is piecewise linear over a partition $\mathcal{P}: 0=t_{0}<t_{1}<$ $\cdots<t_{n+1}=1$, where $\dot{\gamma}_{t}=v_{k} \in \mathbb{R}^{d}$ for $t \in\left[t_{k-1}, t_{k}\right]$. Apparently, the Cartan development $X_{t}$ of $\gamma_{t}$ is a piecewise geodesic. Given $1 \leqslant k \leqslant n$, we have

$$
\dot{X}_{t_{k}-}=\Gamma_{t_{k-1}} \Gamma_{t_{k-1}}^{-1} \Gamma_{t_{k}}\left(\begin{array}{c}
v_{k} \\
0
\end{array}\right)=\Gamma_{t_{k}}\left(\begin{array}{c}
v_{k} \\
0
\end{array}\right),
$$

and

$$
\dot{X}_{t_{k}+}=\Gamma_{t_{k}}\left(\begin{array}{c}
v_{k+1} \\
0
\end{array}\right) \text {. }
$$


Therefore,

$$
\left\langle v_{k}, v_{k+1}\right\rangle_{\text {Euclidean }}=\left\langle\left(\begin{array}{c}
v_{k} \\
0
\end{array}\right),\left(\begin{array}{c}
v_{k+1} \\
0
\end{array}\right)\right\rangle_{*}=\left\langle\dot{X}_{t_{k}-}, \dot{X}_{t_{k}+}\right\rangle_{*},
$$

where the second equality uses that $\Gamma_{t_{k}}$ is an isometry with respect to $\langle\cdot, \cdot\rangle_{*}$ and we define

$$
\left\langle\left(\begin{array}{c}
x_{1} \\
\vdots \\
x_{d+1}
\end{array}\right),\left(\begin{array}{c}
y_{1} \\
\vdots \\
y_{d+1}
\end{array}\right)\right\rangle_{*} \triangleq\left(\begin{array}{c}
x_{1} \\
\vdots \\
x_{d+1}
\end{array}\right) *\left(\begin{array}{c}
y_{1} \\
\vdots \\
y_{d+1}
\end{array}\right)=\sum_{i=1}^{d} x_{i} y_{i}-x_{d+1} y_{d+1} .
$$

This implies that the Cartan development preserves intersection angles.

Fact 2. In a hyperbolic triangle with edges $a, b, c>0$, we have $a \geqslant b+c-$ $\log \frac{2}{1-\cos \theta_{A}}$, where $\theta_{A}$ is the angle opposite $a$.

Proof. The only point which requires some attention is the following fact: for $\lambda>0$, if we consider triangles with the same angle $\theta_{A}$ (its opposite edge being denoted by $a(\lambda))$, and $\lambda b, \lambda c$ being the other two edges, then

$$
f(\lambda) \triangleq \lambda b+\lambda c-a(\lambda)
$$

is monotonely increasing in $\lambda$. Based on this fact, one finds the upper bound of $b+c-a$ to be $\lim _{\lambda \rightarrow \infty}(\lambda b+\lambda c-a(\lambda))$, which can be computed by using the hyperbolic cosine law (c.f. Proof of Lemma 3.4 in [12])

To this end, it suffices to show that $f^{\prime}(\lambda)=b+c-a^{\prime}(\lambda) \geqslant 0$. By the first hyperbolic cosine law, we have

$$
\cosh a(\lambda)=\cosh \lambda b \cosh \lambda c-\sinh \lambda b \sinh \lambda c \cos \theta_{A}
$$

Differentiating with respect to $\lambda$, we obtain that

$$
\begin{aligned}
a^{\prime}(\lambda) \sinh a(\lambda)= & b(\sinh \lambda b \cosh \lambda c-r \cosh \lambda b \sinh \lambda c) \\
& +c(\cosh \lambda b \sinh \lambda c-r \sinh \lambda b \cosh \lambda c)
\end{aligned}
$$

where $r \triangleq \cos \theta_{A}$. For simplicity we write $\sinh =\operatorname{sh}, \cosh =\operatorname{ch}$. Now it suffices to show that

$$
b(\operatorname{sh} \lambda b \cdot \operatorname{ch} \lambda c-r \operatorname{ch} \lambda b \cdot \operatorname{sh} \lambda c)+c(\operatorname{ch} \lambda b \cdot \operatorname{sh} \lambda c-r \operatorname{sh} \lambda b \cdot \operatorname{ch} \lambda c) \leqslant(b+c) \operatorname{sh} a(\lambda) .
$$


We use $X, Y$ to denote the left and right hand sides respectively. From direct computation, we see that

$$
\begin{aligned}
X^{2}= & (b-c r)^{2} \operatorname{sh}^{2} \lambda b \cdot \operatorname{ch}^{2} \lambda c+(c-b r)^{2} \operatorname{ch}^{2} \lambda b \cdot \operatorname{sh}^{2} \lambda c \\
& +\left(2 b c+2 b c r^{2}-2 b^{2} r-2 c^{2} r\right) \operatorname{sh} \lambda b \cdot \operatorname{ch} \lambda b \cdot \operatorname{sh} \lambda c \cdot \operatorname{ch} \lambda c,
\end{aligned}
$$

and by the hyperbolic cosine law (4.5),

$$
\begin{aligned}
Y^{2}= & (b+c)^{2}\left(\left(1+r^{2}\right) \operatorname{sh}^{2} \lambda b \cdot \operatorname{sh}^{2} \lambda c+\operatorname{sh}^{2} \lambda b+\operatorname{sh}^{2} \lambda c\right. \\
& -2 r \operatorname{sh} \lambda b \cdot \operatorname{ch} \lambda b \cdot \operatorname{sh} \lambda c \cdot \operatorname{ch} \lambda c) .
\end{aligned}
$$

By using $\cosh ^{2} x-\sinh ^{2} x=1$, we obtain that

$$
\begin{aligned}
\frac{Y^{2}-X^{2}}{1+r}= & 2 b c(1+r) \operatorname{sh}^{2} \lambda b \cdot \operatorname{sh}^{2} \lambda c-2 b c(1+r) \operatorname{sh} \lambda b \cdot \operatorname{ch} \lambda b \cdot \operatorname{sh} \lambda c \cdot \operatorname{ch} \lambda c \\
& +\left(c^{2}(1-r)+2 b c\right) \operatorname{sh}^{2} \lambda b+\left(b^{2}(1-r)+2 b c\right) \operatorname{sh}^{2} \lambda c .
\end{aligned}
$$

Define $g(r)$ to be the function in $r$ given by the right hand side of the above equality. Then

$$
g(1)=2 b c(\operatorname{sh} \lambda b \cdot \operatorname{ch} \lambda c-\operatorname{ch} \lambda b \cdot \operatorname{sh} \lambda c)^{2} \geqslant 0 .
$$

Moreover,

$$
\begin{aligned}
g^{\prime}(r)= & -2 b c \operatorname{sh} \lambda b \cdot \operatorname{sh} \lambda c \cdot \operatorname{ch} \lambda(b-c) \\
& -c^{2} \operatorname{sh}^{2} \lambda b-b^{2} \operatorname{sh}^{2} \lambda c \\
\leqslant & 0,
\end{aligned}
$$

where the inequality in the final line follows by using $\operatorname{ch} \lambda(b-c) \geqslant 1$ and completing the square. Therefore, $g(r) \geqslant 0$ for $r \in[-1,1]$, which implies that $Y^{2} \geqslant X^{2}$. Since $Y \geqslant 0$, we conclude that $Y \geqslant X$.

Let $\gamma:[0,1] \rightarrow \mathbb{R}^{d}$ be a tree-reduced bounded variation path. From (4.1) and the explicit formula for the Cartan development, it can be shown that (see also (4.9) below), for each $\lambda>0$,

$$
\begin{aligned}
\cosh \rho\left(X_{1}^{\lambda}, o\right) & =\sum_{n=0}^{\infty} \lambda^{2 n} \int_{0<t_{1}<\cdots<t_{2 n}<1}\left\langle\mathrm{~d} \gamma_{t_{1}}, \mathrm{~d} \gamma_{t_{2}}\right\rangle \cdots\left\langle\mathrm{d} \gamma_{t_{2 n-1}}, \mathrm{~d} \gamma_{t_{2 n}}\right\rangle \\
& \leqslant \sum_{n=0}^{\infty} \lambda^{2 n}\left\|\int_{0<t_{1}<\cdots<t_{2 n}<1} \mathrm{~d} \gamma_{t_{1}} \otimes \cdots \otimes \mathrm{d} \gamma_{t_{2 n}}\right\|_{\text {proj }} \quad \text { (see (4.10) below) } \\
& \leqslant \cosh \lambda \widetilde{l} .
\end{aligned}
$$


where $\widetilde{l}$ is defined by (4.3) as the supremum of the (normalized) iterated integrals, $\gamma_{t}^{\lambda} \triangleq \lambda \gamma_{t}(0 \leqslant t \leqslant 1)$ is the path obtained by rescaling $\gamma$ by the factor $\lambda$, and $X_{t}^{\lambda}$ is the hyperbolic development of $\gamma_{t}^{\lambda}$. In particular, we see that $\lambda \widetilde{l} \geqslant \rho\left(X_{1}^{\lambda}, o\right)$.

The previous Fact 2 tells us that for all two-edge piecewise geodesic paths $Y:[0,1] \rightarrow \mathbb{H}^{d}$ with fixed intersection angle $0<\theta<\pi$, the distance between hyperbolic length of $Y$ and $\rho\left(Y_{1}, o\right)$ is uniformly bounded by a constant depending on $\theta$. Now suppose that $\gamma:[0,1] \rightarrow \mathbb{R}^{d}$ is a two-edge piecewise linear path with intersection angle $0<\theta<\pi$. Fact 1 and 2 together implies that

$$
0 \leqslant \lambda\|\gamma\|_{1-\operatorname{var}}-\rho\left(X_{1}^{\lambda}, o\right) \leqslant K(\theta) \triangleq \log \frac{2}{1-\cos \theta}
$$

uniformly in $\lambda>0$. In particular,

$$
\lim _{\lambda \rightarrow \infty} \frac{\rho\left(X_{1}^{\lambda}, o\right)}{\lambda}=\|\gamma\|_{1-\mathrm{var}}
$$

from which we obtain the desired estimate $\tilde{l} \geqslant\|\gamma\|_{1-\text { var. }}$ It is important to note that the angle $\theta$ captures the tree-reduced nature of $\gamma$ in this simple case. Indeed, if $\theta=0, K(\theta)=+\infty$.

With some effort, the previous argument extends to tree-reduced piecewise linear paths with minimal intersection angle given by $\theta>0$. In this case, one can obtain an estimate of the form

$$
0 \leqslant \lambda\|\gamma\|_{1-\mathrm{var}}-\rho\left(X_{1}^{\lambda}, o\right) \leqslant N \cdot \Lambda(\theta)
$$

uniformly in $\lambda>0$, where $N$ is the number of edges of $\gamma$ and $\Lambda(\theta)$ is a constant depending only $\theta$ (which explodes as $\theta \downarrow 0$ ). We again obtain (4.6) and thus the desired estimate. Here $\theta>0$ captures the tree-reduced nature of $\gamma$. With some further delicate analysis, one can establish a similar estimate for a path $\gamma:[0,1] \rightarrow \mathbb{R}^{d}$ which is continuously differentiable when parametrized at constant speed. The estimate takes the form

$$
0 \leqslant \lambda\|\gamma\|_{1-\operatorname{var}}-\rho\left(X_{1}^{\lambda}, o\right) \leqslant C_{1} \lambda\|\gamma\|_{1-\operatorname{var}} \delta_{\gamma}\left(\frac{C_{2}}{\lambda}\right)^{2}
$$

provided that $\lambda$ is large, where $C_{1}, C_{2}$ are universal constants and $\delta_{\gamma}(\cdot)$ is the modulus of continuity for $\dot{\gamma}$. In particular, we again obtain (4.6) and thus the desired estimate. Here the existence of modulus of continuity for the derivative $\dot{\gamma}$ already implies that $\gamma$ is tree-reduced implicitly. In any case, the fundamental 
reason which makes the technique of hyperbolic development work is hidden in the nature of Fact 1 and 2 .

If one is attempting to attack the length conjecture $\widetilde{l}=\|\gamma\|_{1-\text { var }}$ for a general tree-reduced path with bounded variation by using the idea of hyperbolic development, it seems that a crucial point is to find a quantity $\omega_{\gamma}$, a certain kind of "modulus of continuity", which on the one hand captures the tree-reduced nature of $\gamma$ quantitatively, and on the other hand can be used to control the growth of $\lambda \mapsto \lambda\|\gamma\|_{1-\mathrm{var}}-\rho\left(X_{1}^{\lambda}, o\right)$ (difference between hyperbolic length and hyperbolic distance for the rescaled path). Up to the current point, this fascinating and challenging problem remains unsolved.

\subsection{The hyperbolic development of Brownian motion and a lower estimate for $\kappa_{d}$}

In spite of the huge difficulty in obtaining lower estimates of the hyperbolic distance function in the general deterministic setting, it is surprising that a simple martingale argument will give us a meaningful lower estimate for the hyperbolic development of Brownian motion. In particular, we can obtain a lower estimate on the constant $\kappa_{d}$.

From now on, we assume that $\mathbb{R}^{d}$ is equipped with the $l^{p}$-norm for some given $1 \leqslant p \leqslant 2$, and the tensor products over $\mathbb{R}^{d}$ are equipped with the associated projective tensor norms.

The following characterization of projective tensor norms is important for us.

Lemma 4.1. For each $\xi \in\left(\mathbb{R}^{d}\right)^{\otimes n}$, we have

$$
\|\xi\|_{\text {proj }}=\sup \left\{|\Phi(\xi)|: \Phi \in L\left(\mathbb{R}^{d}, \cdots, \mathbb{R}^{d} ; \mathbb{R}^{1}\right),\|\Phi\| \leqslant 1\right\}
$$

where we identify $L\left(\mathbb{R}^{d}, \cdots, \mathbb{R}^{d} ; \mathbb{R}^{1}\right)$ with $\left(\left(\mathbb{R}^{d}\right)^{\otimes n}\right)^{*}$ through the universal property, and

$$
\|\Phi\| \triangleq \inf \left\{C \geqslant 0:\left|\Phi\left(v_{1}, \cdots, v_{n}\right)\right| \leqslant C\left\|v_{1}\right\| \cdots\left\|v_{n}\right\| \quad \forall v_{1}, \cdots, v_{n} \in \mathbb{R}^{d}\right\} .
$$

Proof. See [20], Identity (2.3).

Let $B_{t}=\left(B_{t}^{1}, \cdots, B_{t}^{d}\right)$ be a $d$-dimensional Brownian motion. We define

$$
\widetilde{L}_{t} \triangleq \limsup _{n \rightarrow \infty}\left(\left(\frac{n}{2}\right) !\left\|\mathbb{B}_{0, t}^{n}\right\|_{\text {proj }}\right)^{\frac{2}{n}} .
$$


For each $\lambda>0$, we consider the Cartan development

$$
\left\{\begin{array}{l}
d \Gamma_{t}^{\lambda}=\lambda \Gamma_{t}^{\lambda} F\left(\operatorname{od} B_{t}\right), \quad t \geqslant 0 \\
\Gamma_{0}^{\lambda}=\mathrm{I}_{d+1}
\end{array}\right.
$$

of $\lambda \cdot B_{t}$, where the differential equation is understood in the Stratonovich sense. Let $X_{t}^{\lambda} \triangleq \Gamma_{t}^{\lambda} o$ be the hyperbolic development of $\lambda \cdot B_{t}$. As in the Cartan development driven by bounded variation paths, $\Gamma_{t}^{\lambda}$ also defines a path on the isometry group $\mathrm{SO}(d, 1)$ and hence $X_{t}^{\lambda}$ is a path on $\mathbb{H}^{d}$ starting at $o$.

Picard's iteration again shows that

$$
\Gamma_{t}^{\lambda}=\sum_{n=0}^{\infty} \lambda^{n} \int_{0<t_{1}<\cdots<t_{n}<t} F\left(\operatorname{od} B_{t_{1}}\right) \cdots F\left(\operatorname{od} B_{t_{n}}\right) .
$$

Define $h_{t}^{\lambda} \triangleq\left(X_{t}^{\lambda}\right)^{d+1}$ to be the hyperbolic height of $X_{t}^{\lambda}$ (the last coordinate of $\left.X_{t}^{\lambda}\right)$. It follows from (4.1), (4.8) and the definition of $F$ that

$$
\begin{aligned}
h_{t}^{\lambda} & =\cosh \rho\left(X_{t}^{\lambda}, o\right) \\
& =\sum_{n=0}^{\infty} \lambda^{n} \int_{0<t_{1}<\cdots<t_{n}<t}\left(F\left(\operatorname{od} B_{t_{1}}\right) \cdots F\left(\operatorname{od} B_{t_{n}}\right) o\right)^{d+1} \\
& =\sum_{n=0}^{\infty} \lambda^{2 n} \int_{0<t_{1}<\cdots<t_{n}<t}\left\langle\operatorname{od} B_{t_{1}}, \operatorname{od} B_{t_{2}}\right\rangle \cdots\left\langle\operatorname{od} B_{t_{2 n-1}}, \operatorname{od} B_{t_{2 n}}\right\rangle,
\end{aligned}
$$

The following result shows that the quantity $\widetilde{L}_{t}$ can be controlled from below in terms of the asymptotics of $h_{t}^{\lambda}$ as $\lambda \rightarrow \infty$.

Proposition 4.1. With probability one, we have

$$
\limsup _{\lambda \rightarrow \infty} \frac{1}{\lambda^{2}} \log h_{t}^{\lambda} \leqslant \widetilde{L}_{t}, \quad \forall t \geqslant 0 .
$$

Proof. For each $n \geqslant 1$, define a real-valued $2 n$-linear map $\Phi_{n}$ over $\mathbb{R}^{d}$ by

$$
\Phi_{n}\left(v_{1}, \cdots, v_{2 n}\right) \triangleq\left\langle v_{1}, v_{2}\right\rangle \cdots\left\langle v_{2 n-1}, v_{2 n}\right\rangle, \quad v_{1}, \cdots, v_{2 n} \in \mathbb{R}^{d} .
$$

Since we are taking the $l^{p}$-norm on $\mathbb{R}^{d}$ for $1 \leqslant p \leqslant 2$, we see that

$$
\begin{aligned}
\left|\Phi_{n}\left(v_{1}, \cdots, v_{2 n}\right)\right| & \leqslant\left\|v_{1}\right\|_{l^{2}} \cdots\left\|v_{2 n}\right\|_{l^{2}} \\
& \leqslant\left\|v_{1}\right\|_{l^{p}} \cdots\left\|v_{2 n}\right\|_{l^{p}} .
\end{aligned}
$$


In particular, $\left\|\Phi_{n}\right\| \leqslant 1$. Therefore, by Lemma 4.1 , we have

$$
\left|\int_{0<t_{1}<\cdots<t_{n}<t}\left\langle\operatorname{od} B_{t_{1}}, \operatorname{od} B_{t_{2}}\right\rangle \cdots\left\langle\operatorname{od} B_{t_{2 n-1}}, \operatorname{od} B_{t_{2 n}}\right\rangle\right|=\left|\Phi_{n}\left(\mathbb{B}_{0, t}^{2 n}\right)\right| \leqslant\left\|\mathbb{B}_{0, t}^{2 n}\right\|_{\text {proj }}
$$

Now for each $\alpha \geqslant 1$, define

$$
\widetilde{L}_{t}^{\alpha} \triangleq \sup _{n \geqslant \alpha}\left(\left(\frac{n}{2}\right) !\left\|\mathbb{B}_{0, t}^{n}\right\|_{\text {proj }}\right)^{\frac{2}{n}} .
$$

It follows from (4.7) and (4.8) that

$$
\begin{aligned}
h_{t}^{\lambda} & \leqslant \sum_{n=0}^{\infty} \lambda^{2 n}\left\|\mathbb{B}_{0, t}^{2 n}\right\|_{\text {proj }} \\
& =\sum_{n=0}^{\alpha-1} \lambda^{2 n}\left\|\mathbb{B}_{0, t}^{2 n}\right\|_{\text {proj }}+\sum_{n=\alpha}^{\infty} \lambda^{2 n}\left\|\mathbb{B}_{0, t}^{2 n}\right\|_{\text {proj }} \\
& \leqslant \sum_{n=0}^{\alpha-1} \lambda^{2 n}\left\|\mathbb{B}_{0, t}^{2 n}\right\|_{\text {proj }}+\sum_{n=\alpha}^{\infty} \lambda^{2 n} \cdot \frac{\left(\widetilde{L}_{t}^{2 \alpha}\right)^{n}}{n !} \\
& =\exp \left(\lambda^{2} \widetilde{L}_{t}^{2 \alpha}\right)+\sum_{n=0}^{\alpha-1} \lambda^{2 n}\left(\left\|\mathbb{B}_{0, t}^{2 n}\right\|_{\text {proj }}-\frac{\left(\widetilde{L}_{t}^{2 \alpha}\right)^{n}}{n !}\right) .
\end{aligned}
$$

Therefore,

$$
\begin{aligned}
& \limsup _{\lambda \rightarrow \infty} \frac{1}{\lambda^{2}} \log h_{t}^{\lambda} \\
& \leqslant \limsup _{\lambda \rightarrow \infty} \frac{1}{\lambda^{2}} \log \left(\exp \left(\lambda^{2} \widetilde{L}_{t}^{2 \alpha}\right)+\sum_{n=0}^{\alpha-1} \lambda^{2 n}\left(\left\|\mathbb{B}_{0, t}^{2 n}\right\|_{\text {proj }}-\frac{\left(\widetilde{L}_{t}^{2 \alpha}\right)^{n}}{n !}\right)\right) \\
& =\widetilde{L}_{t}^{2 \alpha} .
\end{aligned}
$$

Since $\alpha$ is arbitrary, we conclude that

$$
\limsup _{\lambda \rightarrow \infty} \frac{1}{\lambda^{2}} \log h_{t}^{\lambda} \leqslant \inf _{\alpha \geqslant 1} \widetilde{L}_{t}^{2 \alpha}=\widetilde{L}_{t} .
$$


The following result is the probabilistic counterpart of a lower estimate on the hyperbolic height function $h_{t}^{\lambda}$.

Lemma 4.2. For any $0<\mu<d-1$, we have

$$
\mathbb{E}\left[\left(h_{t}^{\lambda}\right)^{-\mu}\right] \leqslant \exp \left(-\frac{\lambda^{2} \mu(d-1-\mu)}{2} t\right) .
$$

Proof. Throughout the rest of this paper, we will use $\cdot d$ to denote the Itô differential.

Note that

$$
F\left(\mathrm{~d} B_{t}\right) \cdot F\left(\mathrm{~d} B_{t}\right)=\left(\begin{array}{cc}
0 & \mathrm{~d} B_{t} \\
\left(\mathrm{~d} B_{t}\right)^{*} & 0
\end{array}\right) \cdot\left(\begin{array}{cc}
0 & \mathrm{~d} B_{t} \\
\left(\mathrm{~d} B_{t}\right)^{*} & 0
\end{array}\right)=\left(\begin{array}{cc}
\mathrm{I}_{d} & 0 \\
0 & d
\end{array}\right) \mathrm{d} t .
$$

Applying the Itô-Stratonovich conversion to the differential equation for $\Gamma_{t}^{\lambda}$, we have

$$
\begin{aligned}
\mathrm{d} \Gamma_{t}^{\lambda} & =\lambda \Gamma_{t}^{\lambda} \cdot F\left(\mathrm{~d} B_{t}\right)+\frac{\lambda}{2} d \Gamma_{t}^{\lambda} \cdot F\left(\mathrm{~d} B_{t}\right) \\
& =\lambda \Gamma_{t}^{\lambda} \cdot F\left(\mathrm{~d} B_{t}\right)+\frac{\lambda^{2}}{2} \Gamma_{t}^{\lambda}\left(F\left(\mathrm{~d} B_{t}\right) \cdot F\left(\mathrm{~d} B_{t}\right)\right) \\
& =\lambda \Gamma_{t}^{\lambda} \cdot F\left(\mathrm{~d} B_{t}\right)+\frac{\lambda^{2}}{2} \Gamma_{t}^{\lambda}\left(\begin{array}{cc}
\mathrm{I}_{d} & 0 \\
0 & d
\end{array}\right) \mathrm{d} t .
\end{aligned}
$$

Therefore by restricting our attention to the $(d+1, d+1)$ coordinate of the matrix,

$$
\begin{aligned}
\mathrm{d} h_{t}^{\lambda} & =\mathrm{d}\left(\Gamma_{t}^{\lambda}\right)_{d+1}^{d+1} \\
& =\lambda \sum_{i=1}^{d}\left(\Gamma_{t}^{\lambda}\right)_{i}^{d+1} \cdot \mathrm{d} B_{t}^{i}+\frac{\lambda^{2} d}{2} h_{t}^{\lambda} \mathrm{d} t .
\end{aligned}
$$

Moreover, since $\Gamma_{t}^{\lambda} \in \mathrm{SO}(d, 1)$, we know that

$$
\sum_{i=1}^{d}\left(\left(\Gamma_{t}^{\lambda}\right)_{i}^{d+1}\right)^{2}-\left(h_{t}^{\lambda}\right)^{2}=-1
$$

and hence by (4.11) and (4.12),

$$
\mathrm{d} h_{t}^{\lambda} \cdot \mathrm{d} h_{t}^{\lambda}=\lambda^{2} \sum_{i=1}^{d}\left(\left(\Gamma_{t}^{\lambda}\right)_{i}^{d+1}\right)^{2} \mathrm{~d} t=\lambda^{2}\left(\left(h_{t}^{\lambda}\right)^{2}-1\right) \mathrm{d} t .
$$


Note that $h_{t}^{\lambda} \geqslant 1$ and hence we may apply Itô's formula to $\left(h_{t}^{\lambda}\right)^{-\mu}$ and then (4.11) to obtain,

$$
\begin{aligned}
\mathrm{d}\left(h_{t}^{\lambda}\right)^{-\mu}= & -\mu\left(h_{t}^{\lambda}\right)^{-(\mu+1)} \cdot \mathrm{d} h_{t}^{\lambda}+\frac{\mu(\mu+1)}{2}\left(h_{t}^{\lambda}\right)^{-(\mu+2)}\left(\mathrm{d} h_{t}^{\lambda} \cdot \mathrm{d} h_{t}^{\lambda}\right) \\
= & -\lambda \mu\left(h_{t}^{\lambda}\right)^{-(\mu+1)} \sum_{i=1}^{d}\left(\Gamma_{t}^{\lambda}\right)_{i}^{d+1} \cdot \mathrm{d} B_{t}^{i} \\
& -\left(\frac{\lambda^{2} \mu(d-1-\mu)}{2}\left(h_{t}^{\lambda}\right)^{-\mu}+\frac{\lambda^{2} \mu(\mu+1)}{2}\left(h_{t}^{\lambda}\right)^{-(\mu+2)}\right) \mathrm{d} t .
\end{aligned}
$$

By taking expectation and differentiating with respect to $t$, we obtain that

$$
\begin{aligned}
\frac{\mathrm{d}}{\mathrm{d} t} \mathbb{E}\left[\left(h_{t}^{\lambda}\right)^{-\mu}\right]= & -\frac{\lambda^{2} \mu(d-1-\mu)}{2} \mathbb{E}\left[\left(h_{t}^{\lambda}\right)^{-\mu}\right] \\
& -\frac{\lambda^{2} \mu(\mu+1)}{2} \mathbb{E}\left[\left(h_{t}^{\lambda}\right)^{-(\mu+2)}\right] \\
\leqslant & -\frac{\lambda^{2} \mu(d-1-\mu)}{2} \mathbb{E}\left[\left(h_{t}^{\lambda}\right)^{-\mu}\right],
\end{aligned}
$$

where in the final inequality we used that $h_{t}^{\lambda} \geqslant 1$ (see for example (4.9)). By Gronwall's inequality, we arrive at

$$
\mathbb{E}\left[\left(h_{t}^{\lambda}\right)^{-\mu}\right] \leqslant \exp \left(-\frac{\lambda^{2} \mu(d-1-\mu)}{2} t\right) .
$$

Now we can state our main lower estimate on $\kappa_{d}$.

Theorem 4.1. Under the $l^{p}$-norm $(1 \leqslant p \leqslant 2)$ on $\mathbb{R}^{d}$ and the associated projective tensor norms on the tensor products, we have

$$
\kappa_{d} \geqslant \frac{d-1}{2}
$$

Proof. Fix $t>0, \lambda>0$ and $0<\mu<d-1$. According to Lemma 4.2 (which we have just proved), for each $K>0$,

$$
\begin{aligned}
\mathbb{P}\left(h_{t}^{\lambda} \leqslant K\right) & =\mathbb{P}\left(\left(h_{t}^{\lambda}\right)^{-\mu} \geqslant K^{-\mu}\right) \\
& \leqslant K^{\mu} \mathbb{E}\left[\left(h_{t}^{\lambda}\right)^{-\mu}\right] \\
& \leqslant K^{\mu} \exp \left(-\frac{\lambda^{2} \mu(d-1-\mu)}{2} t\right) .
\end{aligned}
$$


Let $s \in \mathbb{Q}$ such that $s<t$. Now for each $m \geqslant 1$, define $\lambda_{m} \triangleq m$ and

$$
K_{m} \triangleq \exp \left(\frac{m^{2}(d-1-\mu)}{2} s\right)
$$

It follows that

$$
\mathbb{P}\left(h_{t}^{\lambda_{m}} \leqslant K_{m}\right) \leqslant \exp \left(-\frac{m^{2} \mu(d-1-\mu)}{2}(t-s)\right) .
$$

In particular, $\sum_{m=1}^{\infty} \mathbb{P}\left(h_{t}^{\lambda_{m}} \leqslant K_{m}\right)<\infty$. By the Borel-Cantelli lemma, there exists a $\mathbb{P}$-null set $\mathcal{N}(s, t, \mu)$, such that for any $\omega \notin \mathcal{N}(s, t, \mu)$, there exists $M(\omega) \geqslant$ 1 with

$$
h_{t}^{\lambda_{m}}(\omega)>\exp \left(\frac{m^{2}(d-1-\mu)}{2} s\right), \quad \forall m \geqslant M(\omega) .
$$

Therefore,

$$
\limsup _{m \rightarrow \infty} \frac{1}{m^{2}} \log h_{t}^{\lambda_{m}}(\omega) \geqslant \frac{d-1-\mu}{2} s .
$$

By enlarging the $\mathbb{P}$-null set through rationals $s \uparrow t$ and $\mu \downarrow 0$, we conclude that

$$
\limsup _{m \rightarrow \infty} \frac{1}{m^{2}} \log h_{t}^{\lambda_{m}} \geqslant \frac{d-1}{2} t
$$

for almost surely.

Finally, according to Proposition 4.1 which relates $\tilde{L}_{t}$ and $h_{t}$, we obtain that

$$
\kappa_{d}=\frac{\widetilde{L}_{t}}{t} \geqslant \frac{d-1}{2} .
$$

\section{Applications to the Brownian rough path}

We present a few interesting consequences of the lower estimate on $\kappa_{d}$ given in Theorem 4.1.

Let us consider the $d$-dimensional Brownian motion $B_{t}$ on $[0,1]$. Recall that with probability one, $B_{t}$ has a canonical lifting $\mathbf{B}_{t}$ as geometric $p$-rough path for $2<p<3$. As a process on $G^{2}\left(\mathbb{R}^{d}\right)$, the Brownian rough path $\mathbf{B}_{t}$ is canonically defined and it is independent of the choice of tensor norms on $\left(\mathbb{R}^{d}\right)^{\otimes 2}$. 
Corollary 5.1. If $d>1$, then for almost every $\omega$, the path $t \mapsto \mathbf{B}_{t}(\omega)$ is treereduced.

Proof. Let the tensor products be equipped with the projective tensor norms associated with the $l^{2}$-norm on $\mathbb{R}^{d}$. From Proposition 3.3, for every $\omega$ outside some $\mathbb{P}$-null set $\mathcal{N}$,

$$
\widetilde{L}_{s, t}(\omega)=\kappa_{d}(t-s) \quad \forall s<t .
$$

In addition, from Theorem 4.1 we know that the constant $\kappa_{d}$ is strictly positive and hence $\tilde{L}_{s, t}>0$ for $s<t$. This implies that for every $\omega \notin \mathcal{N}$, the signature of $\mathbf{B}(\omega), \mathbb{B}_{s, t}$, is non-trivial for all $s<t$, which according to [3] is the definition of $\mathbf{B}$ being tree-reduced.

It was first proved by Le Jan and Qian [14] (see also [2]) that the Stratonovitch signature of Brownian sample paths determine the samples paths almost surely. Using Corollary 5.1, we obtain a stronger result below which explicitly reconstruct the sample paths as well as its parametrization.

Corollary 5.2. If $d>1$, then with probability one, Brownian rough path together with its parametrization can be recovered from its signature.

Proof. We define an equivalence relation $\sim$ on rough paths so that $\mathbf{X} \sim \mathbf{Y}$ if and only if there is a continuous, strictly increasing, onto function $\sigma$ from $[0,1]$ onto $[0,1]$ such that

$$
\mathbf{X}_{t}=\mathbf{Y}_{\sigma(t)}
$$

(i.e. $\mathbf{X}$ and $\mathbf{Y}$ are reparametrization of each other. This relation was considered in depth in [2], Section 5.3).

Lemma 4.6 in [3] states that two tree-reduced geometric rough path have the same signature if and only if they are in the same equivalence class. Corollary 5.1 therefore implies that for any two $\omega_{1}$ and $\omega_{2}$ outside some $\mathbb{P}$-null set $\mathcal{N}, \mathbf{B}\left(\omega_{1}\right)$ and $\mathbf{B}\left(\omega_{2}\right)$ have the same signature if and only if they are in the same equivalence class.

Pick an arbitrary representative $\left(\mathbf{X}_{t}\right)_{0 \leqslant t \leqslant 1} \in[\mathbf{B}(\omega)]$. Then

$$
\mathbf{X}_{t}=\mathbf{B}_{\sigma(t)}(\omega), \quad 0 \leqslant t \leqslant 1
$$

for some unique reparametrization $\sigma$ that we want to figure out. According to Proposition 3.3, we have

$$
\sigma(t)=\frac{1}{\kappa_{d}} \limsup _{n \rightarrow \infty}\left(\left(\frac{n}{2}\right) !\left\|\mathbb{X}_{0, t}^{n}\right\|_{\text {proj }}\right)^{\frac{2}{n}}
$$


where we again choose the projective tensor norms on the tensor products associated with the $l^{2}$-norm on $\mathbb{R}^{d}$. (Recall that by Theorem $4.1, \kappa_{d} \neq 0$ ) The underlying path $\mathbf{B}(\omega)$ is then given by

$$
\mathbf{B}_{t}(\omega)=\mathbf{X}_{\sigma^{-1}(t)}, \quad 0 \leqslant t \leqslant 1
$$

Another way of understanding the previous result is the following. Since $[\mathbf{B}(\omega)]$ can be recovered from its signature, we know that the image of the signature path $\mathbb{B}(\omega)$ can be recovered from its endpoint. For every tensor element $\xi=$ $\left(1, \xi_{1}, \xi_{2}, \cdots\right)$ which can be realized as the signature of some Brownian sample path, we then have

$$
\mathbf{B}_{\|\xi\| / \kappa_{d}}(\omega)=\pi^{(2)}(\xi)
$$

where

$$
\|\xi\| \triangleq \limsup _{n \rightarrow \infty}\left(\left(\frac{n}{2}\right) !\left\|\xi_{n}\right\|_{\text {proj }}\right)^{\frac{2}{n}}
$$

and $\pi^{(2)}: T\left(\left(\mathbb{R}^{d}\right)\right) \rightarrow T^{(2)}\left(\left(\mathbb{R}^{d}\right)\right)$ is the canonical projection map.

Beyond the study of signature, Corollary 5.1 also gives the following property of Brownian rough path, which we are unable to find in the literature.

Corollary 5.3. There exists a $\mathbb{P}$-null set $\mathcal{N}$, such that for any two distinct $\omega_{1}, \omega_{2} \notin$ $\mathcal{N}, \mathbf{B}\left(\omega_{1}\right)$ and $\mathbf{B}\left(\omega_{2}\right)$ cannot be equal up to a reparametrization.

Proof. We follow the same notation as in the proof of Corollary 5.1. Given two distinct $\omega_{1}, \omega_{2} \notin \mathcal{N}$, suppose that

$$
\mathbf{B}_{t}\left(\omega_{2}\right)=\mathbf{B}_{\sigma(t)}\left(\omega_{1}\right), \quad 0 \leqslant t \leqslant 1,
$$

for some reparametrization $\sigma:[0,1] \rightarrow[0,1]$. Then the signature of $t \rightarrow \mathbf{B}_{\sigma(t)}$ is equal to $S(\mathbf{B})_{\sigma(t)}$ (see Lemma 1.4 in [4]) we have

$$
\widetilde{L}_{0, \sigma(t)}\left(\omega_{1}\right)=\kappa_{d} \sigma(t)
$$

and

$$
\widetilde{L}_{0, t}\left(\omega_{2}\right)=\kappa_{d} t
$$

But from assumption we know that $\widetilde{L}_{0, \sigma(t)}\left(\omega_{1}\right)=\widetilde{L}_{0, t}\left(\omega_{2}\right)$. Therefore, we must have $\sigma(t)=t$ and hence $\mathbf{B}\left(\omega_{1}\right)=\mathbf{B}\left(\omega_{2}\right)$. 


\section{Further remarks and related problems}

In Theorem 1.1, we considered the tail asymptotics of the Brownian signature defined in terms of iterated Stratonovich's integrals. Stratonovich's integrals arise naturally when we study Brownian motion from the rough path point of view. On the other hand, one could ask a similar question for Itô's iterated integrals. Indeed, if we define

$$
\widehat{L}_{s, t} \triangleq \limsup _{n \rightarrow \infty}\left(\left(\frac{n}{2}\right) !\left\|\int_{s<u_{1}<\cdots<u_{n}<t} \mathrm{~d} B_{u_{1}} \otimes \cdots \otimes \mathrm{d} B_{u_{n}}\right\|_{l^{1}}\right)^{\frac{2}{n}}
$$

where the iterated integrals are defined in the sense of Itô and the tensor products are equipped with the $l^{1}$-norm, then by a similar type of arguments, one can show that for each $s<t$

$$
\frac{d(t-s)}{2} \leqslant \widehat{L}_{s, t} \leqslant \frac{d^{2}(t-s)}{2}
$$

almost surely. Since the lifting of Brownian motion in Itô's sense is not a geometric rough path, uniqueness of signature result does not apply and the intrinsic meaning of the quantity $\widehat{L}_{s, t}$ is unclear. The proof of (6.2) will not be included here since it is essentially parallel to the Stratonovich case.

Our main result of Theorem 1.1 gives rise to many interesting and related problems in the probabilistic context.

(1) The first interesting and immediate question one could come up with is the exact value of $\kappa_{d}$ and its probabilistic meaning. In view of the length conjecture (1.4) and Theorem 1.1, if we consider the projective tensor norms on the tensor products induced by the Euclidean norm on $\mathbb{R}^{d}$, it is quite natural to expect that, $\kappa_{d}$ would have a meaning related to certain kind of quadratic variation for the Brownian rough path. It also seems that there are rooms for improving the upper estimate for $\kappa_{d}$. The point is that in the proof of Lemma 3.1, if we shuffle an arbitrary long word $\left\{i_{1}, \cdots, i_{n}\right\}$ over $\{1, \cdots, d\}$ with itself, the chance of hitting a nonzero coefficient in the $2 n$-degree component of the Brownian expected signature is quite small. But to make the analysis precise, some hard combinatorics argument for the shuffle product structure might be involved.

(2) If $\kappa_{d}$ is related to certain kind of quadratic variation for the Brownian motion, it is reasonable to expect that our main result and corollaries apply to diffusions or even general continuous semimartingales, though there is no reason to believe that in this case the corresponding $\widetilde{L}_{s, t}$ will still be deterministic. For Gaussian processes, it is even not clear that any analogous version of $\widetilde{L}_{s, t}$ would 
be meaningful since for instance we know that

$$
\lim _{n \rightarrow \infty} \sum_{i=1}^{n}\left|B_{\frac{i}{n}}-B_{\frac{i-1}{n}}\right|^{p}=0 \text { or } \infty
$$

in probability for a fractional Brownian motion with Hurst parameter $H \in(0,1)$, according to whether $p H>1$ or $p H<1$.

(3) There is a quite subtle point in the discussion of Section 6 . With probability one, the lifting map $\omega \mapsto \mathbf{B}(\omega)$ is canonically well-defined. Therefore, although Corollary 5.2 (the uniqueness result) is stated at the level of the Brownian rough path, by projection to degree one, it also holds at the level of sample paths.

However, it is not at all clear if the first part of Corollary 5.3 is true at the level of Brownian sample paths. More precisely, to our best knowledge, a solution to the following classical question for Brownian motion is not known (at least not to us yet): does there exist a $\mathbb{P}$-null set $\mathcal{N}$, such that no two sample paths outside $\mathcal{N}$ can be equal up to a non-trivial reparametrization? This question is stated for Brownian sample paths and has nothing to do with the lifting of Brownian motion to rough paths.

It is a subtle point that the result of Corollary 5.3 does not yield an affirmative answer easily to the above question. Indeed, if one wants to apply Corollary 5.3, a missing point is whether the lifting operation and the reparametrization operation are commutative outside some universal $\mathbb{P}$-null set. In other words, it is not known if there exists a $\mathbb{P}$-null set $\mathcal{N}$, such that one could define a lifting map $\omega \mapsto \mathbf{B}(\omega)$ for all $\omega \notin \mathcal{N}$, which satisfies

$$
\text { B. }\left(\omega_{\sigma}\right)=\mathbf{B}_{\sigma(\cdot)}(\omega)
$$

for all reparametrizations $\sigma:[0,1] \rightarrow[0,1]$. When defining the almost sure lifting of Brownian motion, the $\mathbb{P}$-null set comes with the given choice of approximation. It is quite subtle (and could be false) to see if the $\mathbb{P}$-null set can be chosen in a universal way.

(4) A final remark is about whether the limsup in (6.1) can be replaced by sup. This is true for bounded variations, the proof of which we now briefly explain.

Let $g=\left(1, g_{1}, g_{2}, \cdots\right)$ be a group-like element. From the shuffle product formula,

$$
g_{k}^{\otimes n}=\sum_{\sigma \in \mathcal{S}(k, \cdots, k)} \mathcal{P}^{\sigma}\left(g_{n k}\right)
$$

Therefore,

$$
\left\|g_{k}\right\|_{\text {proj }}^{n} \leqslant \frac{(n k) !}{(k !)^{n}}\left\|g_{n k}\right\|_{\text {proj }}
$$


It follows that

$$
\left(k !\left\|g_{k}\right\|_{\text {proj }}\right)^{\frac{1}{k}} \leqslant\left((n k) !\left\|g_{n k}\right\|_{\text {proj }}\right)^{\frac{1}{n k}}, \quad \forall n, k \geqslant 1 .
$$

Therefore, we conclude that

$$
\sup _{n \geqslant 1}\left(n !\left\|g_{n}\right\|_{\text {proj }}\right)^{\frac{1}{n}}=\limsup _{n \rightarrow \infty}\left(n !\left\|g_{n}\right\|_{\text {proj }}\right)^{\frac{1}{n}}
$$

This is indeed true for any given admissible norms. A similar statement with a fractional factorial normalization (which naturally corresponds to the rough path situation) is not true. Indeed, considering the Brownian motion case, we have

$$
\sup _{n \geqslant 1}\left(\left(\frac{n}{2}\right) !\left\|\mathbb{B}_{0,1}^{n}\right\|_{\text {proj }}\right)^{\frac{2}{n}} \geqslant\left(\left(\frac{1}{2}\right) !\left\|B_{1}-B_{0}\right\|_{\mathbb{R}^{d}}\right)^{2},
$$

while on the other hand, by Theorem 3.1,

$$
\limsup _{n \rightarrow \infty}\left(\left(\frac{n}{2}\right) !\left\|\mathbb{B}_{0,1}^{n}\right\|_{\text {proj }}\right)^{\frac{2}{n}}=\kappa_{d}
$$

for almost surely. Therefore, with positive probability the "sup" is not equal to the "limsup" for the Brownian signature.

\section{References}

[1] G. Ben Arous, Flots et series de Taylor stochastiques, Probab. Theory Related Fields 81: 29-77, 1989.

[2] H. Boedihardjo and X. Geng, The uniqueness of signature problem in the non-Markov setting, Stochastic Process. Appl. 125 (12): 4674-4701, 2015.

[3] H. Boedihardjo, X. Geng, T. Lyons and D. Yang, The signature of a rough path: Uniqueness, Adv. Math. 293: 720-737, 2016.

[4] H. Boedihardjo, X. Geng, T. Lyons, D. Yang, Note on the signatures of rough paths in a Banach space, arXiv:1501.04172, 2015.

[5] J.W. Cannon, W..J. Floyd, R. Kenyon and W.R. Parry, hyperbolic geometry, in Flavors of Geometry, Math. Sci. Res. Inst. Publ. 31: 59-115, Cambridge University Press, Cambridge, 1997. 
[6] M. Caruana, T. Lévy and T. Lyons, Differential equations driven by rough paths, Springer, 2007.

[7] J. Chang, N. Duffield, H. Ni and W. Xu, Signature inversion for monotone paths, Electron. Commun. Probab. 22 (42): 1-11, 2017.

[8] K.T. Chen, Iterated integrals and exponential homomorphisms, Proceedings of the London Mathematical Society 3(1), 502-512, 1954.

[9] T. Fawcett, Problems in stochastic analysis: connections between rough paths and non-commutative harmonic analysis, Ph.D. thesis, University of Oxford, 2003.

[10] P. Friz and N. Victoir, Multidimensional stochastic processes as rough paths, Cambridge University Press, 2010.

[11] X. Geng, Reconstruction for the signature of a rough path, Proc. Lond. Math. Soc. 114 (3): 495-526, 2017.

[12] B. Hambly onsand T. Lyons, Uniqueness for the signature of a path of bounded variation and the reduced path group, Ann. of Math. 171 (1): 109-167, 2010 .

[13] K. Hara and M. Hino, Fractional order Taylor's series and the neo-classical inequality, Bull. Lond. Math. Soc. 42, 467-477, 2010.

[14] Y. Le Jan and Z. Qian, Stratonovich's signatures of Brownian motion determine Brownian sample paths, Probab. Theory Relat. Fields 157: 209-223, 2013.

[15] T. Lyons, Differential equations driven by rough signals, Rev. Mat. Iberoamericana 14 (2), 215-310, 1998.

[16] T. Lyons and Z. Qian, System control and rough paths, Oxford University Press, 2002.

[17] T. Lyons and Nicolas Victoir, Cubature on Wiener space, Proceedings of the Royal Society of London A: Mathematical, Physical and Engineering Sciences 460(2041), 169-198, 2004.

[18] T. Lyons and W. Xu, Hyperbolic development and the inversion of signature, J. Funct. Anal. 272 (7): 2933-2955, 2015. 
[19] T. Lyons and W. Xu, Inverting the signature of a path, to appear in J. Eur. Math. Soc., 2017.

[20] R. Ryan, Introduction to Tensor Products of Banach Spaces, Springer, 2016.

[21] E. M. Sipiläinen, A pathwise view of solutions of stochastic differential equations, Ph.D. thesis, University of Edinburgh, 1993.

[22] A. Ursitti, Computation of some transcendental integrals from path signatures, arXiv:1601:03135, 2016.

[23] W. Yang, L. Jin, and M. Liu, Chinese character-level writer identification using path signature feature, DropStroke and deep CNN, Document Analysis and Recognition (ICDAR), 2015 13th International Conference on, IEEE, 2015. 